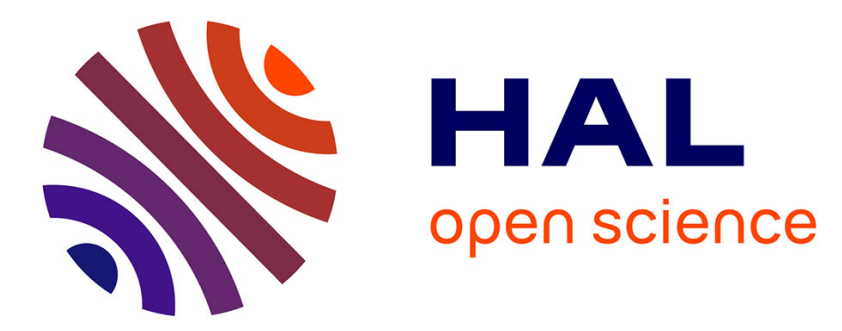

\title{
Observers are Unnecessary for Output-Feedback Control of Lagrangian Systems
}

Antonio Loria

\section{To cite this version:}

Antonio Loria. Observers are Unnecessary for Output-Feedback Control of Lagrangian Systems. IEEE Transactions on Automatic Control, 2016, 61 (4), pp.905-920. 10.1109/TAC.2015.2446831 . hal01357249

\section{HAL Id: hal-01357249 https://hal.science/hal-01357249}

Submitted on 6 Sep 2016

HAL is a multi-disciplinary open access archive for the deposit and dissemination of scientific research documents, whether they are published or not. The documents may come from teaching and research institutions in France or abroad, or from public or private research centers.
L'archive ouverte pluridisciplinaire HAL, est destinée au dépôt et à la diffusion de documents scientifiques de niveau recherche, publiés ou non, émanant des établissements d'enseignement et de recherche français ou étrangers, des laboratoires publics ou privés. 


\title{
Observers are Unnecessary for Output-Feedback Control of Lagrangian Systems
}

\author{
Antonio Loria
}

\begin{abstract}
We address and solve some long-standing yet welldocumented open problems on output feedback tracking control of Euler-Lagrange systems with arbitrarily high relative degree -this includes underactuated systems. Our main contribution is to establish a theoretical foundation for the use of so-called dirty derivatives, a common "ad-hoc" replacement of unavailable state measurements such as generalized velocities, whence obviating the the use of observers for the purpose of position-feedback tracking control. Reminiscent of passivity-based control for robot manipulators, our control law is globally Lipschitz and the controller dynamics is linear. For relative-degree-two fullyactuated Lagrangian systems without dissipative forces (friction) and with bounded inertia matrix we establish uniform global asymptotic stability in closed loop. Furthermore, we show that our control approach applies to Lagrangian systems augmented by a chain of integrators (relative degree $m+2$ systems). The design method, which is based on a recursive procedure in the spirit of backstepping control, is intuitive as it exploits structural properties such as passivity and inherent input-output stability. As a corollary, we solve an output feedback global-tracking control problem for flexible-joint robots but also for systems coupled with output-feedback linearizable actuator dynamics. In addition, we discuss remaining open problems of fairly general interest in the realm of analysis and design of robust nonlinear systems.
\end{abstract}

\section{INTRODUCTION}

We study lossless Euler-Lagrange systems, defined by

$$
D(q) \ddot{q}+C(q, \dot{q}) \dot{q}+g(q)=u
$$

where $q \in \mathbb{R}^{n}$ denotes the generalized positions, $\dot{q}$ denotes the generalized velocities, $D: \mathbb{R}^{n} \rightarrow \mathbb{R}^{n \times n}$ denotes the inertia matrix function, which satisfies $D(q)=D(q)^{\top}, C: \mathbb{R}^{n} \times$ $\mathbb{R}^{n} \rightarrow \mathbb{R}^{n \times n}$ is the Coriolis and centrifugal forces matrix function, $g: \mathbb{R}^{n} \rightarrow \mathbb{R}^{n}$ represents the vector of forces which are derived from the potential energy function $U: \mathbb{R}^{n} \rightarrow \mathbb{R}$ i.e., $g(q):=\frac{\partial U}{\partial q}(q)$ and $u \in \mathbb{R}^{n}$ is the vector of control inputs. All functions are assumed to be sufficiently smooth.

We revisit the problem of output-feedback tracking control, which consists in designing a dynamic controller with output $u$, using $q$ as the only plant measurement and ensuring that, given a smooth bounded trajectory $t \mapsto q_{d}$, the generalized coordinates satisfy

$$
\lim _{t \rightarrow \infty} q(t) \rightarrow q_{d}(t), \quad \lim _{t \rightarrow \infty} \dot{q}(t) \rightarrow \dot{q}_{d}(t) .
$$

More precisely (and of much higher difficulty) we establish uniform global asymptotic stability of the origin of the closedloop system. We put special emphasis on the qualifier 'global'

Preliminary version: arXiv:1307.4659 [math.OC, cs.SY] available at http://arxiv.org/abs/1307.4659

The author is with the CNRS, France. Address: LSS-CentraleSupelec, 3 Rue Joliot-Curie, 91192 Gif-sur-Yvette, France. loria@lss.supelec. fr which implies that the property must hold for all initial states of the closed-loop system in the Euclidean space of the latter, that is, including the tracking errors and the dynamic controller's states. This property is not to be confused with inappropriate terminologies such as "global on the set $X \subset \mathbb{R}^{n}$ " or the weaker property "global in the plant's variables and semi-global in the controller's" $-c f$., e.g., [1].

Establishing uniform global asymptotic stability for (1) along time-varying trajectories (tracking control) is a difficult problem that cannot be overestimated. Actually, as it is nicely showed in the seminal article [2], it lies at the edge of the achievable. Roughly, from the main results in [2] it may be concluded that the system

$$
d \ddot{q}+c \dot{q}^{2}=u, \quad q, u \in \mathbb{R}
$$

cannot be stabilized globally via dynamic feedback of $q$ only. The obstacle is that the system does not possess the unboundedness observability (concept introduced in this reference) property from $q$ that is, the solution $\dot{q}(t)$ may escape to infinity even for bounded values of $q(t)$. However, Lagrangian systems escape to this impediment in view of the structural property that the matrix $\dot{D}(q)-2 C(q, \dot{q})$ is skew-symmetric.

Since at least the early results of Nicosia and coauthors - see e.g., [3] as well as many posterior references by these authors, in the last 25 years or so there have been numerous attempts to solve the nicknamed "global-tracking-control" problem of Lagrangian systems. A common trend in the literature is to design observer-based controllers and to employ Lypunov's direct method to establish global stability. However, finding a strict Lyapunov function -see [4], is a major challenge wich remains open for general $n>1$ degree-of-freedom systems. At least, this is the case for lossless systems (1) since, otherwise, a simple and effective way to obtain negative terms of $|\dot{q}|$ in the bound on $\dot{V}$, is to impose the practically reasonable, but theoretically conservative, assumption that the system possesses natural viscous friction. See, e.g., [5] in which the authors use the Lagrangian model

$$
D(q) \ddot{q}+C(q, \dot{q}) \dot{q}+F \dot{q}+g(q)=u
$$

where $F=F^{\top}>0$.

To the best of our knowledge the only article that presents a dynamic output-feedback controller for lossless EulerLagrange systems (in generalized coordinates) together with a strict Lyapunov function, albeit for one-degree-of-freedom systems, is [6]. In the latter we presented a dynamic output feedback controller which employs nonlinear corrective terms of exponential growth and we established uniform global asymptotic stability of the origin of the closed-loop system. 
Unfortunately, this method seems to apply only to one-degreeof-freedom systems

$$
d(q) \ddot{q}+c(q) \dot{q}^{2}+g(q)=u,
$$

with the property $\partial d(q) / \partial q=2 c(q)$. The extension of the results in [6] to the case of $n$-degree-of-freedom systems has eluded several authors -see e.g., [1], [7]. The controller in the former is guaranteed (in the non-adaptive case, only) to achieve uniform asymptotic stability for any system's initial conditions provided that the controller's trajectories belong to a forward-invariant set. Moreover, the result in [1] relies on the assumption that the model includes viscous friction (of known magnitude in the non-adaptive case) -see Eq. (2), and that the forces derived from the potential energy are bounded. The controller of [7] is given in implicit form hence it is not implementable without velocity measurements.

In [8] the author employs an approximate-differentiation filter to construct a dynamic position feedback controller. Using Tychonov's theorem for singularly-perturbed systems it is showed that uniform asymptotic stability may be rendered global provided that the unique pole of the filter is placed at $-\infty$. In other words, the result in [8] actually guarantees semi-global asymptotic stability. As a matter of fact, the same property may be established via Lyapunov's direct method see e.g., [9]. The controllers from [8], [9] are direct extensions of a set-point controller that was independently published in [10] and [11].

The obvious difficulty to design observers for (1) is the nonlinearity in the unmeasured variables $\dot{q}$. Hence, a natural alternative approach is to seek a coordinate transformation which facilitates observer design. Such methods typically rely on the ability to factorize the inertia matrix $D(q)$ in closed form, see e.g., [12] where conditions are given to diagonalize the inertia matrix. In a similar train of thought the seminal work of G. Besançon -see e.g., [13], is to be emphasized, along with the more recent works [14], [15], [16], [17].

In [18], whose main ideas may be traced back to [13], a controller is designed for one-degree-of-freedom systems, based on the factorization of the inertia term $d(q)$. This results in a system affine in $\dot{q}$ and a controller of linear growth. As an aside, the controller in [18] yields a much superior performance to that from [6] which employs terms of exponential growth. Unfortunately, the extension of the control method in [18] to the case of $n$-degree-of-freedom systems is stymied by structural properties; a fact well studied in [19]. Interesting exceptions for which appropriate changes of coordinates apply to particular classes of Euler-Lagrange systems include [20], [21] and some references therein.

More recently, in [15] was developed a set-point controller for underactuated Hamiltonian systems, upon the ideas laid in [16]. Based on the factorization method from the last two references, in [22] (which is a byproduct of [17]) a global result for tracking control of Hamiltonian systems was presented. The controller relies on a closed-form factorization of $D(q)^{-1}$ and a clever but intricate observer design due to [23].

As far as we know, [24] and [22], which appeared during the initial preparation of this paper, were the first articles in which uniform global asymptotic stability for $n$-degrees-of-freedom systems is established. Actually, in [22], [17] uniform global exponential stability (in the space of the transformed system) is established via Lyapunov's direct method for the case that the inertia matrix is bounded and uniform global asymptotic stability holds in the case that the inertia matrix is not bounded from above. This is in contrast with the literature, including the present paper, where boundedness of $D$ is typically assumed. See also [20] where exponential stability is established for a class of Euler-Lagrange systems.

This paper contains several contributions, the most important of which is to provide a theoretical foundation for the use of approximate differentiation. Conceptually, the latter consists in using a "filtered" version of unmeasured velocities. Such a filter is implemented using positions only but by no means it may be considered as an observer or a velocity estimator.

In a first part of the paper we revise the controller independently proposed in [8], [9] and [25] for tracking control of lossless Euler-Lagrange systems (1). Strictly speaking, we establish uniform global asymptotic stability for the origin of the closed-loop system. In contrast to [24] we provide explicit simple and intuitive conditions on the control gains. In contrast to [8], [9], [25] we establish a global result. With respect to [22], and any other observer-based result for that effect, our controller does not rely on the estimation of the unmeasured variables. Moreover, the control law is globally Lipschitz, the controller is linear and all equations and conditions are expressed in Lagrangian coordinates. This makes our first main statement, Theorem 1, self-contained and particularly concise.

In a second part of the paper, we show how the control law may be implemented through a chain of integrators via a recursive control design, reminiscent of backstepping control, in which the derivatives of virtual control inputs are replaced by approximate differentiation filters. That is, we solve the output feedback control problem for arbitrarily-highrelative-degree systems (with respect to generalized positions). This includes the output feedback control problem for underactuated systems hence, as a corollary of our main results, we recover a statement on output feedback control for flexible joint manipulators: we establish uniform global asymptotic stability without measurement of link velocities.

The rest of the paper is organized as follows. For the sake of clarity, in Section II we recall basic stability definitions. In Section III we present our first result, for systems with relative degree two, such as fully-actuated Lagrangian systems. In Section IV we present the extension of our main results to the case of higher-relative-degree systems. In Section V we discuss remaining related open problems and, finally, we provide some concluding remarks in Section VI.

\section{Preliminaries}

Notation. Recall that a continuous function $\alpha: \mathbb{R}_{\geq 0} \rightarrow \mathbb{R}_{\geq 0}$ is of class $\mathcal{K}$ if it is strictly increasing and $\alpha(\overline{0})=0$, a continuous function $\sigma: \mathbb{R}_{\geq 0} \rightarrow \mathbb{R}_{\geq 0}$ is of class $\mathcal{L}$ if it is strictly decreasing and $\sigma(s) \rightarrow 0$ as $s \rightarrow \infty$; a continuous function $\beta: \mathbb{R}_{\geq 0} \times \mathbb{R}_{\geq 0} \rightarrow \mathbb{R}_{\geq 0}$ is of class $\mathcal{K} \mathcal{L}$ if $\beta(r, \cdot) \in \mathcal{L}$ 
and $\beta(\cdot, s) \in \mathcal{K}$; a continuous function $\alpha: \mathbb{R}_{\geq 0} \rightarrow \mathbb{R}_{\geq 0}$ is of class $\mathcal{K}_{\infty}$ if $\alpha \in \mathcal{K}$ and $\alpha(s) \rightarrow \infty$ as $s \rightarrow \infty$. We denote by $|\cdot|$, the Euclidean norm of vectors (or any other compatible norm) and the induced norm of matrices.

To remove all possible ambiguity it seems fitting to recall, from [26], a few definitions of stability and some statements that are either known or are re-stated in an original manner, for the purposes of this paper. Consider the dynamic system

$$
\dot{x}=f(t, x), \quad x \in \mathbb{R}^{n}, t \in \mathbb{R}_{\geq 0}
$$

where $f$ satisfies the conditions for local existence and uniqueness of solutions and $f(t, 0) \equiv 0$. We denote by $x\left(\cdot, t_{\circ}, x_{\circ}\right)$, or when the context is clear by $x(\cdot)$, the solutions of (4) with initial times $t_{\circ} \in \mathbb{R}_{\geq 0}$ and initial states $x_{\circ} \in \mathbb{R}$ that is, we have $x\left(t_{\circ}, t_{\circ}, x_{\circ}\right)=x_{\circ}$.

Definition 1 (Uniform global boundedness) The solutions of (4) are said to be uniformly globally bounded if there exist $\gamma \in \mathcal{K}_{\infty}$ and $c>0$ such that, for all $\left(t_{\circ}, x_{\circ}\right) \in \mathbb{R}_{\geq 0} \times \mathbb{R}^{n}$, the solution $x\left(\cdot, t_{\circ}, x_{\circ}\right)$ satisfies

$$
\left|x\left(t, t_{\circ}, x_{\circ}\right)\right| \leq \gamma\left(\left|x_{\circ}\right|\right)+c \quad \forall t \geq t_{\circ} .
$$

Theorem 1 Let $V: \mathbb{R}_{\geq 0} \times \mathbb{R}^{n} \rightarrow \mathbb{R}_{\geq 0}$ be continuously differentiable; let $\alpha_{1}, \alpha_{2}$ be functions of class $\mathcal{K}_{\infty}$ and let $a, c \in \mathbb{R}$ be such that $c>0$ and

$$
\begin{array}{cc}
\alpha_{1}(|x|) \geq V(t, x) \geq \alpha_{2}(|x|)+a & \forall(t, x) \in \mathbb{R}_{\geq 0} \times \mathbb{R}^{n} \\
\dot{V}(t, x):=\frac{\partial V}{\partial t}+\frac{\partial V}{\partial x} f(t, x) \leq 0 & \forall t \in \mathbb{R}_{\geq 0}, x:|x| \geq c
\end{array}
$$

Then, the solutions of (4) are uniformly globally bounded.

Although unusual in "modern" literature, the following fundamental definition may be found, for instance, in [26].

Definition 2 (Uniform global stability) The origin of system (4) is said to be uniformly globally stable if there exists $\gamma \in$ $\mathcal{K}_{\infty}$ such that for each $\left(t_{\circ}, x_{\circ}\right) \in \mathbb{R}_{\geq 0} \times \mathbb{R}^{n}$, each solution $x\left(\cdot, t_{\circ}, x_{\circ}\right)$ satisfies

$$
\left|x\left(t, t_{\circ}, x_{\circ}\right)\right| \leq \gamma\left(\left|x_{\circ}\right|\right) \quad \forall t \geq t_{\circ} .
$$

Note that uniform global stability is tantamount to uniform stability plus uniform global boundedness.

Theorem 2 Let the conditions of Theorem 1 hold for $a=c=$ 0 . Then, the origin of (4) is uniformly globally stable. If the conditions hold only in an open neighborhood of the origin with $\alpha_{1}, \alpha_{2} \in \mathcal{K}$, the origin is uniformly stable.

Definition 3 (Uniform global attractivity) The origin of system (4) is said to be uniformly globally attractive if for each $r, \sigma>0$ there exists $T>0$ such that

$$
\left|x_{\circ}\right| \leq r \Longrightarrow\left|x\left(t, t_{\circ}, x_{\circ}\right)\right| \leq \sigma \quad \forall t \geq t_{\circ}+T .
$$

\section{Definition 4 (Uniform Global Asymptotic Stability)}

The origin of system (4) is said to be uniformly globally asymptotically stable if it is

- uniformly stable;

- the solutions are uniformly globally bounded;

- the origin is uniformly globally attractive.
It is important to emphasize that only together the three conditions in Definition 4 imply the existence of a class $\mathcal{K} \mathcal{L}$ function $\beta$ such that the solutions of (4) satisfy

$$
|x(t)| \leq \beta\left(\left|x_{\circ}\right|, t-t_{\circ}\right) \quad \forall t \geq t_{\circ} \geq 0 .
$$

The latter leads to the construction of converse uniformly monotone Lyapunov functions and, in turn, implies robustness with respect to external perturbations. Such bound cannot be obtained if any of the three properties in Definition 4 fails. In particular, uniform global asymptotic stability may not be concluded either from uniform stability plus uniform global attractivity alone -see [27]; whence the importance of uniform global boundedness in nonlinear time-varying systems.

The following theorem, which corresponds to [28, Lemma 2], establishes uniform global asymptotic stability without a strict Lyapunov function, in the spirit of integral criteria such as Barbalăt's lemma.

Theorem 3 Let $F: \mathbb{R}_{\geq 0} \times \mathbb{R}^{n} \rightarrow \mathbb{R}^{n}$ be continuous such that $F(\cdot, 0) \equiv 0$. Let $\{x=0\}$ be uniformly globally stable. If, moreover, there exists a continuous positive definite function $\Upsilon: \mathbb{R}^{n} \rightarrow \mathbb{R}_{\geq 0}$ and, for each pair of positive numbers $(r, \tilde{v})$, there exists $\beta_{r \tilde{v}}>0$ such that, for all $\left(t_{\circ}, x_{\circ}\right) \in \mathbb{R}_{\geq 0} \times B_{r}$, all solutions $x\left(\cdot, t_{0}, x_{\circ}\right)$ satisfy

$$
\int_{t_{\circ}}^{t}\left[\Upsilon\left(x\left(\tau, t_{\circ}, x_{\circ}\right)\right)-\tilde{v}\right] d \tau \leq \beta_{r \tilde{v}}
$$

for all $t \geq t_{0}$, then the origin is uniformly globally asymptotically stable.

Remark 1 Ineq. (8) guarantees uniform global attractivity. Roughly speaking, (8) implies that the trajectories are integrable, modulo $\Upsilon(\cdot)$, out of any ball of radius depending on $\tilde{v}$. This implies that $\Upsilon(x(t))$ converges to the interior of such ball in finite time. Since $\tilde{v}$ is arbitrary, we may conclude that (7) holds for any $\sigma>0$. See [28] for rigorous proof.

\section{RELATIVE-DEGREE-2 SYSTEMS}

In this section we address the output feedback tracking control problem for systems with relative degree two with respect to the generalized positions $q$. This is the case of fullyactuated mechanical systems, such as robot manipulators with rigid joints.

We make a standing assumption that is fairly standard in the literature of robot control but which is also satisfied by a number of Euler-Lagrange systems, such as electrical and electro-mechanical -see [29], as well as some marine vehicles -see [30]. A complete characterization of the class of systems that satisfy this hypothesis is provided in [31], [32].

\section{Assumption 1}

1) There exist positive real numbers $d_{m}$ and $d_{M}$ such that

$$
d_{m} \leq|D(q)| \leq d_{M}, \quad \forall q \in \mathbb{R}^{n} ;
$$

2) there exists $k_{c}>0$ such that

$$
\begin{array}{ll}
|C(x, y)| \leq k_{c}|y| & \forall x, y \in \mathbb{R}^{n}, \\
C(x, y) z=C(x, z) y & \forall x, y, z \in \mathbb{R}^{n} ;
\end{array}
$$

3) the matrix $\dot{D}(q)-2 C(q, \dot{q})$ is skew symmetric. 
Remark 2 The boundedness assumption on the inertia matrix is little conservative; for instance, it holds for (but it is not limited to) robot manipulators composed of revolute joints only or prismatic joints only. See [31] for a complete characterization. Nonetheless, the main result in [22], [17] does not rely on uniform boundedness of $D$; as it is illustrated through a simple example in [33], this is an important relaxation.

The control problem that we solve in this paper is stated as follows.

\section{Definition 5 (global output-feedback tracking control)}

Consider the EL system (1) under Assumption 1. Furthermore, suppose that only position measurements are available, that the given reference trajectory $t \mapsto q_{d}$ is of class $\mathrm{C}^{2}$ and that there exists $k_{\delta}>0$ such that

$$
\max \left\{\sup _{t \geq 0}\left|q_{d}(t)\right|, \sup _{t \geq 0}\left|\dot{q}_{d}(t)\right|, \sup _{t \geq 0}\left|\ddot{q}_{d}(t)\right|\right\} \leq k_{\delta} .
$$

Under these conditions, find a dynamic output-feedback controller

$$
\begin{aligned}
\dot{q}_{c} & =f\left(t, q_{c}, q\right) \\
u & =u\left(t, q_{c}, q\right)
\end{aligned}
$$

such that the closed-loop system

$$
\begin{gathered}
D(q) \ddot{q}+C(q, \dot{q}) \dot{q}+g(q)=u\left(t, q_{c}, q\right) \\
\dot{q}_{c}=f\left(t, q_{c}, q\right)
\end{gathered}
$$

has a unique equilibrium at

$$
\begin{gathered}
\left(\tilde{q}, \dot{\tilde{q}}, q_{c}-q_{c}^{*}\right)=(0,0,0), \\
\tilde{q}:=q-q_{d}(t), \quad \dot{\tilde{q}}:=\dot{q}-\dot{q}_{d}(t)
\end{gathered}
$$

where $q_{c}^{*}$ is a solution to (12) subject to $q \equiv q_{d}$ and this equilibrium is uniformly globally asymptotically stable.

Our first theorem improves the main result in [24] which solves the long-standing open problem defined above -cf. [22].

Theorem 4 Consider the system (1) under Assumption 1. Let $a, b, k_{p}$ and $k_{d}$ be positive constants, let

$$
a>1, \quad \frac{k_{d}}{16} \frac{b}{a}>k_{c} k_{\delta}
$$

and consider the dynamic position-feedback controller

$$
\begin{aligned}
\dot{q}_{c} & =-a\left(q_{c}+b \tilde{q}\right) \\
\vartheta & =q_{c}+b \tilde{q} \\
u & =-k_{p} \tilde{q}-k_{d} \vartheta+D(q) \ddot{q}_{d}+C\left(q, \dot{q}_{d}\right) \dot{q}_{d}+g(q)
\end{aligned}
$$

where $q_{d}$ is described in Definition 5. Then, the origin $\{z=0\}$ with $z:=\left[\begin{array}{lll}\tilde{q}^{\top} & \dot{\tilde{q}}^{\top} & q_{c}^{\top}\end{array}\right]^{\top}$ is uniformly globally asymptotically stable.

Remark 3 In the statement of Theorem 4 we use scalar gains $a, b, k_{p}$ and $k_{d}$ merely for clarity of exposition; the result holds if the gains are diagonal positive matrices by replacing condition (14) with

$$
\frac{k_{d_{m}}}{16} \frac{b_{m}}{a_{M}}>k_{c} k_{\delta}
$$

where $(\cdot)_{m}$ and $(\cdot)_{M}$ denote, respectively, the smallest and largest elements in the diagonal of $(\cdot)$.
The controller (15) is based on its "set-point controller" counter-part, first published in [10] and subsequently used by many other authors. Indeed, (15) is reminiscent of the controller in [8] and it corresponds verbatim to that from [9] -see also [25], where semi global uniform asymptotic stability is also established under much more stringent conditions than (14). This condition is also in great contrast with those imposed in [22], [17] which appeared at the time of the initial submission of this paper -see also [24], [34].

Even though the controller in Theorem 4, per se, is not original, the extent of the statement can hardly be overestimated: 1) Theorem 4 obviates the use of observers. To circumvent the lack of velocity measurements, the controller employs a widely-used ad hoc alternative to differentiation. Indeed, Eqs. (15a), (15b) correspond to the state-space representation of the so-called dirty-derivatives filter

$$
\vartheta=\frac{b}{s+a} \dot{\tilde{q}} \quad \Leftrightarrow \quad \vartheta=\frac{b s}{s+a} \tilde{q}
$$

whose output is commonly used in control practice to replace the unavailable velocities $\dot{\tilde{q}}$. The system in (16) is not an observer.

2) Globality is achieved via a dynamic controller of striking simplicity: the controller dynamics is linear and autonomous while its output, the control law $u(t, \tilde{q}, \dot{\tilde{q}}, \vartheta)$, is bounded in $t$ and $\tilde{q}$ and globally-Lipschitz uniformly in $t$. Moreover, the sole tuning rule, (14), is meaningful; it imposes a very natural constraint on the damping gain $k_{d}$, scaled by the filter's DC gain, $b / a$. The factor $(1 / 16)$ is not tight.

3) Purely from a dynamical-systems perspective, Theorem 4 establishes for (1), (15) the strongest property desirable for a nonlinear time-varying system: uniform global asymptotic stability. This solves a problem open for more than 25 years. As a matter of fact, achieving such property for nonlinear timevarying systems with non-globally Lipschitz nonlinearities of the unmeasured variables is at the edge of the achievable via dynamic output feedback $-c f$. [2] and Section V.

\section{Proof of Theorem 4}

The closed-loop equation is obtained by replacing $u$ from (15c) in (1) and adding $-C\left(q, \dot{q}_{d}\right) \dot{q}+C(q, \dot{q}) \dot{q}_{d}=0$ to the right-hand side of (15c) hence,

$$
D(q) \ddot{\tilde{q}}+\left[C(q, \dot{q})+C\left(q, \dot{q}_{d}\right)\right] \dot{\tilde{q}}+k_{p} \tilde{q}+k_{d} \vartheta=0 .
$$

Then, for the purpose of analysis, we differentiate (15b) and use (15a) to obtain

$$
\dot{\vartheta}=-a \vartheta+b \dot{\tilde{q}}
$$

The point $\{x=0\}$ where $x:=\left[\tilde{q}^{\top} \dot{\tilde{q}}^{\top} \vartheta^{\top}\right]^{\top}$ is an equilibrium of (17), (18) and is unique. Then, a direct computation shows that $\{z=0\}$ is a unique equilibrium of the closed-loop equations (15a), (17). Also, $\{x=0\}$ is uniformly globally asymptotically stable for (17), (18) if and only if so is $\{z=0\}$ for the closed-loop equations (15a), (17). Therefore, we proceed to analyze the stability of (17), (18) at the origin, $\{x=0\}$. This analysis is divided in four ordered steps in which we establish: 
(1) uniform forward completeness -see Lemma 1 below;

(2) uniform global boundedness -see Lemma 2, farther down;

(3) uniform stability -this is contained in the subsequent Lemma 3 however, it follows trivially via Lyapunov's first method and it is also implicitly contained in the proof of the main result in [9].

(4) uniform global attractivity -this is the main statement of Lemma 3.

Lemma 1 Under the conditions of Theorem 4 the closedloop system (1), (15) is uniformly forward complete; moreover, there exist $c_{1}, c_{2}>0$ such that

$$
|x(t)| \leq c_{1}\left|x\left(t_{\circ}\right)\right| e^{c_{2}\left(t-t_{\circ}\right)} \quad \forall t \geq t_{\circ} \geq 0 .
$$

Proof: Consider the Lyapunov function candidate $V_{1}$ : $\mathbb{R}_{\geq 0} \times \mathbb{R}^{3 n} \mapsto \mathbb{R}_{\geq 0}$ defined as

$$
V_{1}(t, \tilde{q}, \dot{\tilde{q}}, \vartheta)=\frac{1}{2}\left(\dot{\tilde{q}}^{\top} D\left(\tilde{q}+q_{d}(t)\right) \dot{\tilde{q}}+k_{p}|\tilde{q}|^{2}+\frac{k_{d}}{b}|\vartheta|^{2}\right)
$$

which, under Assumption 1, satisfies

$$
\begin{gathered}
\alpha_{1}|x|^{2} \geq V_{1}(t, \tilde{q}, \dot{\tilde{q}}, \vartheta) \geq \alpha_{2}|x|^{2} \\
\alpha_{1}:=\frac{1}{2} \max \left\{d_{M}, k_{p}, \frac{k_{d}}{b}\right\}, \alpha_{2}:=\frac{1}{2} \min \left\{d_{m}, k_{p}, \frac{k_{d}}{b}\right\} .
\end{gathered}
$$

Furthermore, using $\dot{D}(q)=C(q, \dot{q})+C(q, \dot{q})^{\top}$, we see that the total derivative of $V_{1}$ along the closed-loop trajectories of (17), (18), satisfies

$$
\begin{aligned}
\dot{V}_{1} & =-\frac{k_{d} a}{b}|\vartheta|^{2}+\dot{\tilde{q}}^{\top} C\left(q, \dot{q}_{d}\right) \dot{\tilde{q}} \\
& \leq-\frac{k_{d} a}{b}|\vartheta|^{2}+k_{c} k_{\delta}|\dot{\tilde{q}}|^{2} .
\end{aligned}
$$

Therefore, $\dot{V}_{1} \leq k_{c} k_{\delta}|x|^{2}$ and, defining $v_{1}(t) \quad:=$ $V_{1}(t, \tilde{q}(t), \dot{\tilde{q}}(t), \vartheta(t))$, we obtain $\dot{v}_{1}(t) \leq\left(k_{c} k_{\delta} / \alpha_{2}\right) v_{1}(t)$. The statement follows after integrating the latter, defining

$$
c_{1}:=\sqrt{\frac{\alpha_{1}}{\alpha_{2}}}, \quad c_{2}:=\frac{k_{c} k_{\delta}}{2 \alpha_{2}}
$$

and invoking the comparison principle.

Lemma 2 Under the conditions of Theorem 4, the closedloop trajectories of the system (1), (15) are uniformly globally bounded.

Proof: We analyze the solutions to (17), (18) with initial conditions $t_{\circ} \geq 0$ and $x\left(t_{\circ}\right)=x_{\circ} \in B_{r}$ where $r>0$ is arbitrarily fixed. The proof follows by reductio ad absurdum. We establish that if the solutions grow unboundedly, $V_{1}$ "becomes" non-increasing along trajectories, for a sequence of sufficiently large time instances.

More precisely, assume that $|\dot{\tilde{q}}(t)| \rightarrow \infty$ as $t \rightarrow \infty$; according to Lemma $1,|\dot{\tilde{q}}(t)|$ satisfies (19). Now, let $\mathcal{J}:=\left\{t_{i}\right\}$ be a divergent sequence of 'large' time instances i.e., $t_{i} \rightarrow \infty$ as $i \rightarrow \infty, t_{1} \geq t_{\circ}+T$ with $T \gg 1$. Let $\mathcal{J}$ generate another divergent sequence $\left\{\dot{\tilde{q}}\left(t_{i}\right)\right\} \succ 0$. More specifically, assume that there exists at least one $k \leq n$ and a sequence $\mathcal{J}$ such that for every $t_{i} \in \mathcal{J}$ we have $\dot{\tilde{q}}_{k}\left(t_{i}\right) \geq 0$. As it shall become clear below, there is no loss of generality in assuming divergence to $+\infty$ since the same reasoning applies for any sequence $\left\{\dot{\tilde{q}}_{k}\left(t_{i}\right)\right\} \prec 0$ strictly decreasing to $-\infty$. Also, note that there is no particular assumption regarding the divergent behaviour of $|\dot{\tilde{q}}(t)|$; in particular, the sequence $\left\{\dot{\tilde{q}}\left(t_{i}\right)\right\} \succ 0$ may be constructed even if $|\dot{\tilde{q}}(t)|$ diverges in an oscillatory manner. Furthermore, oscillations of unboundedly increasing frequency are excluded since the origin is uniformly asymptotically stable -see the proof of Lemma 3 and [9].

By continuity of solutions, for each element of $\left\{t_{i}\right\}$, and for each $k \leq n$ such that $\left\{\dot{\tilde{q}}_{k}\left(t_{i}\right)\right\}$ is strictly increasing, let us define on a $\Delta$-neighbourhood of $t_{i}$, with $\Delta>0$, an absolutely continuous non-decreasing function $\nu_{i k}:\left[t_{i}-\Delta, t_{i}\right] \rightarrow \mathbb{R}_{\geq 0}$ as

$$
\nu_{i k}(t):=\dot{\tilde{q}}_{k}(t) \quad \forall t \in\left[t_{i}-\Delta, t_{i}\right]
$$

Next, let

$$
\delta_{i k}(t):=\dot{\tilde{q}}_{k}(t)-\nu_{i k}\left(t_{i}\right), \quad t \in\left[t_{i}-\Delta, t_{i}\right]
$$

the function $\delta_{i k}$ is absolutely continuous, strictly increasing, $\delta_{i k}\left(t_{i}\right)=0$ and $\delta_{i k}(t) \leq 0$ for all $t \in\left[t_{i}-\Delta, t_{i}\right]$.

On the other hand, the solution of (18), for any $k \leq n$, $t_{\circ} \geq 0$ and $t \geq t_{\circ}$, corresponds to

$$
\vartheta_{k}(t)=\vartheta_{k}\left(t_{\circ}\right) e^{-a\left(t-t_{\circ}\right)}+b \int_{t_{\circ}}^{t} e^{-a(t-\tau)} \dot{\tilde{q}}_{k}(\tau) d \tau
$$

therefore, defining $\vartheta_{i k}^{-}=\vartheta_{k}\left(t_{i}-\Delta\right)$, we obtain,

$$
\begin{aligned}
\vartheta_{k}(t)= & \vartheta_{i k}^{-} e^{-a\left(t-t_{i}+\Delta\right)}+b \nu_{i k}\left(t_{i}\right) \int_{t_{i}-\Delta}^{t} e^{-a(t-\tau)} d \tau \\
& -b \int_{t_{i}-\Delta}^{t}\left|\delta_{i k}(\tau)\right| e^{-a(t-\tau)} d \tau \quad \forall t \in\left[t_{i}-\Delta, t_{i}\right] .
\end{aligned}
$$

By the definition of $\delta_{i k}$ and $\nu_{i k}$, for sufficiently large $t_{i}$, $\nu_{i k}\left(t_{i}\right) \geq 2 \delta_{M}$ where $\delta_{M}=\max \left\{\delta_{i k}(t)\right\}$ for all $t \in$ $\left[t_{i}-\Delta, t_{i}\right], t_{i} \geq 0$-as a matter of fact, it has been showed in [35] that there exists such $\delta_{M}$ independent of $t_{i}$ - hence,

$$
\vartheta_{k}\left(t_{i}\right) \geq \frac{b}{2 a} \nu_{i k}\left(t_{i}\right)\left[1-e^{-a \Delta}\right]+\vartheta_{i k}^{-} e^{-a \Delta}
$$

and

$$
\frac{\nu_{i k}\left(t_{i}\right)}{\vartheta_{k}\left(t_{i}\right)} \leq \frac{2 a}{b\left[1-e^{-a \Delta}\right]}\left[1-\frac{\vartheta_{i k}^{-} e^{-a \Delta}}{\vartheta_{k}\left(t_{i}\right)}\right] .
$$

In view of the non-decreasing nature of $\left\{\vartheta_{k}\left(t_{i}\right)\right\}$ the factor in brackets on the right-hand side of (23) is positive and smaller than one for any $\Delta>0$ and sufficiently large $t_{i}$ hence, by setting $\Delta \geq \ln 2 / a<1$-see (14), we obtain

$$
\frac{\nu_{i k}\left(t_{i}\right)}{\vartheta_{k}\left(t_{i}\right)}<\frac{4 a}{b} \text {. }
$$

On the other hand, in the limit, as $\Delta \rightarrow 0$, we have $\nu_{i k}\left(t_{i}\right) / \vartheta_{k}\left(t_{i}\right) \leq 2(a / b)$.

The inequality (24) holds for any pair of link coordinates $\left(\vartheta_{k}, \dot{\tilde{q}}_{k}\right)$ and any $k \leq n$ but not necessarily with the same sequence $\mathcal{J}:=\left\{t_{i}\right\}$. For each link, i.e., for each $k \leq n$, let us denote by $\mathcal{J}_{k}:=\left\{{ }^{k} t_{i}\right\}$ the sequence of times which generates a divergent sequence $\left\{\dot{\tilde{q}}_{k}\left({ }^{k} t_{i}\right)\right\} \rightarrow+\infty$ for which the previous computations hold, up to (24). Next, consider the sequence $\widetilde{\mathcal{J}}:=\left\{\tau_{i}\right\}$ formed by the union of all the latter i.e.,

$$
\widetilde{\mathcal{J}}:=\mathcal{J}_{1} \cup \mathcal{J}_{2} \cup \cdots \mathcal{J}_{k} \cdots \cup \mathcal{J}_{n} .
$$


On the other hand, defining $v_{1}(t):=V_{1}(t, \tilde{q}(t), \dot{\tilde{q}}(t), \vartheta(t))$, it follows from (22) that, for any $t$,

$$
\dot{v}_{1}(t) \leq-\frac{k_{d} a}{b}|\vartheta(t)|^{2}+k_{c} k_{\delta}|\dot{\tilde{q}}(t)|^{2}
$$

hence, the latter also holds for $t=\tau_{i}$ for any $\tau_{i} \in \widetilde{\mathcal{T}}$. It follows that, for any subsequence $\left\{\tau_{i}^{\prime}\right\} \subseteq \widetilde{\mathcal{J}}$ such that $\left\{\left|q\left(\tau_{i}^{\prime}\right)\right|\right\} \rightarrow+\infty$ we have, from (24),

$$
\begin{aligned}
\dot{v}_{1}\left(\tau_{i}^{\prime}\right) & \leq-\frac{k_{d} a}{b}\left|\vartheta\left(\tau_{i}^{\prime}\right)\right|^{2}+k_{c} k_{\delta}\left|\vartheta\left(\tau_{i}^{\prime}\right)\right|^{2} \frac{\left|\nu_{i}\left(\tau_{i}^{\prime}\right)\right|^{2}}{\left|\vartheta\left(\tau_{i}^{\prime}\right)\right|^{2}} \\
& \leq-\left[\frac{k_{d} a}{b}-\frac{16 k_{c} k_{\delta} a^{2}}{b^{2}}\right]\left|\vartheta\left(\tau_{i}^{\prime}\right)\right|^{2}
\end{aligned}
$$

where we introduced $\nu_{i}:=\left[\nu_{i 1} \ldots \nu_{i n}\right]^{\top}$. In view of (14) we have $\dot{v}_{1}\left(\tau_{i}^{\prime}\right) \leq 0$ which implies that the sequence $\left\{v_{1}\left(\tau_{i}^{\prime}\right)\right\}$ is non-increasing. Since $V_{1}$ is positive definite and proper, it follows that that the sequence $\left\{\left|x\left(\tau_{i}^{\prime}\right)\right|\right\}$ is bounded. This contradicts the initial assumption that $\left\{\left|\dot{\tilde{q}}\left(t_{i}\right)\right|\right\}$ grows unboundedly and implies, in turn, that $\left\{\left|\vartheta\left(t_{i}\right)\right|\right\}$ is also bounded, since the filter (16) has finite gain. By Lemma 1, $|\dot{\tilde{q}}(t)|$ and $|\vartheta(t)|$ are also bounded on every interval $\left[t_{i}, t_{i+1}\right]$ hence, from uniform forward completeness, we conclude that $|\dot{\tilde{q}}(t)|$ and $|\vartheta(t)|$ are uniformly bounded for all $t$.

The previous arguments also apply (with appropriate modifications) to any infinite sequence $\left\{t_{i}\right\}$ generating a strictly decreasing sequence $\left\{\dot{\tilde{q}}\left(t_{i}\right)\right\} \prec 0$.

Now we prove that $|\tilde{q}(t)|$ is also bounded. Let it be otherwise and consider the (twice differentiable) function $V_{2}: \mathbb{R}_{\geq 0} \times \mathbb{R}^{3 n} \rightarrow \mathbb{R}_{\geq 0}$,

$$
V_{2}(t, \tilde{q}, \dot{\tilde{q}}, \vartheta)=\left(\varepsilon_{1} \tilde{q}-\varepsilon_{2} \vartheta\right)^{\top} D\left(\tilde{q}+q_{d}(t)\right) \dot{\tilde{q}}, \quad \varepsilon_{1}, \quad \varepsilon_{2}<1
$$

which, in view of (17) and (18), satisfies

$$
\begin{aligned}
\dot{V}_{2}= & \left(\varepsilon_{1} \tilde{q}-\varepsilon_{2} \vartheta\right)^{\top}\left(-k_{d} \vartheta-k_{p} \tilde{q}-\left[C(q, \dot{q})+C\left(q, \dot{q}_{d}\right)\right] \dot{\tilde{q}}\right) \\
& +\varepsilon_{1} \dot{\tilde{q}}^{\top} D(q) \dot{\tilde{q}}-\varepsilon_{2}(-a \vartheta+b \dot{\tilde{q}})^{\top} D(q) \dot{\tilde{q}} \\
& +\left(\varepsilon_{1} \tilde{q}-\varepsilon_{2} \vartheta\right)^{\top} \dot{D}(q) \dot{\tilde{q}} .
\end{aligned}
$$

Let $R$ be an arbitrary positive number and define

$$
\Omega:=\left\{x \in \mathbb{R}^{3 n}: \tilde{q} \in \mathbb{R}^{n}, \max \{|\dot{\tilde{q}}|,|\vartheta|\} \leq R\right\} .
$$

Then,

$$
\begin{aligned}
& \left|\varepsilon_{1} \tilde{q}^{\top} C(q, \dot{q})^{\top} \dot{\tilde{q}}\right| \leq \varepsilon_{1} k_{c}|\tilde{q}||\dot{\tilde{q}}|\left[R+k_{\delta}\right] \\
& \left|\varepsilon_{2} \vartheta^{\top} C(q, \dot{q})^{\top} \dot{\tilde{q}}\right| \leq \varepsilon_{2} k_{c}|\vartheta||\dot{\tilde{q}}|\left[R+k_{\delta}\right]
\end{aligned}
$$

-see (11), which implies that for all $x \in \Omega$, all the terms of undefined sign on the right-hand side of (27) may be upper bounded by a first-order polynomial of $|\tilde{q}|$. Therefore, using Assumption 1 and (28), we see that there exist positive numbers $c_{1}, c_{2}$ such that $v_{2}(t):=V_{2}(t, \tilde{q}(t), \dot{\tilde{q}}(t), \vartheta(t))$ satisfies

$$
\dot{v}_{2}(t) \leq-\varepsilon_{1} k_{p}|\tilde{q}(t)|^{2}+c_{1}|\tilde{q}(t)|+c_{2}
$$

for all $t \geq t_{\circ}$ and $x(t) \in \Omega$. Furthermore, let $V: \mathbb{R}_{\geq 0} \times \mathbb{R}^{3 n} \rightarrow$ $\mathbb{R}_{\geq 0}$ be defined as

$$
V(t, x):=V_{1}(t, \tilde{q}, \dot{\tilde{q}}, \vartheta)+V_{2}(t, \tilde{q}, \dot{\tilde{q}}, \vartheta)
$$

which is positive definite and proper under the conditions of Theorem 4 and we show that its total derivative along trajectories becomes negative for large values of $|\tilde{q}(t)|$. To that end, let us define

$$
\begin{gathered}
M_{1}(t, x):=\left[\begin{array}{cc}
k_{p} I & 2 \varepsilon_{1} D\left(\tilde{q}+q_{d}(t)\right) \\
2 \varepsilon_{1} D\left(\tilde{q}+q_{d}(t)\right)^{\top} & D\left(\tilde{q}+q_{d}(t)\right)
\end{array}\right] \\
M_{2}(t, x):=\left[\begin{array}{cc}
\frac{k_{d}}{b} I & -2 \varepsilon_{2} D\left(\tilde{q}+q_{d}(t)\right) \\
-2 \varepsilon_{2} D\left(\tilde{q}+q_{d}(t)\right)^{\top} & D\left(\tilde{q}+q_{d}(t)\right)
\end{array}\right],
\end{gathered}
$$

so we have

$$
V(t, x) \geq \frac{1}{4}\left[\begin{array}{c}
\tilde{q} \\
\tilde{\tilde{q}}
\end{array}\right]^{\top} M_{1}(t, x)\left[\begin{array}{c}
\tilde{q} \\
\dot{\tilde{q}}
\end{array}\right]+\frac{1}{4}\left[\begin{array}{c}
\vartheta \\
\dot{\tilde{q}}
\end{array}\right]^{\top} M_{2}(t, x)\left[\begin{array}{c}
\vartheta \\
\dot{\tilde{q}}
\end{array}\right]
$$

and both $M_{1}(t, x)$ and $M_{2}(t, x)$ are positive definite (uniformly in $t$ ) respectively if

$$
\frac{k_{p}}{4 d_{M}}>\varepsilon_{1}^{2}, \quad \frac{k_{d}}{4 b d_{M}}>\varepsilon_{2}^{2} .
$$

For any given positive gains $k_{p}, k_{d}$ and $b$ and the constant $d_{M}$ one can always find $\varepsilon_{1}, \varepsilon_{2}>0$ such that the inequalities in (31) hold. Moreover, from Assumption 1.1 we also see that $V$ is drecrescent; indeed, we have

$$
V(t, x) \leq\left[\begin{array}{c}
\tilde{q} \\
\tilde{\tilde{q}}
\end{array}\right]^{\top} M_{1}(t, x)\left[\begin{array}{c}
\tilde{q} \\
\tilde{\tilde{q}}
\end{array}\right]+\left[\begin{array}{c}
\vartheta \\
\dot{\tilde{q}}
\end{array}\right]^{\top} M_{2}(t, x)\left[\begin{array}{c}
\vartheta \\
\dot{\tilde{q}}
\end{array}\right]
$$

where the induced norms of $M_{1}$ and $M_{2}$ are uniformly bounded from above, due to (9).

Now, using (22) on $v_{1}(t)$ as well as (29) we see that $v(t):=$ $V(t, x(t))$ satisfies

$$
\dot{v}(t) \leq-\varepsilon_{1} k_{p}|\tilde{q}(t)|^{2}+c_{1}|\tilde{q}(t)|+c_{2}+k_{c} k_{\delta} R^{2}
$$

for all $x(t) \in \Omega, t \geq t_{\circ} \geq 0$. We see that $\dot{v}(t) \leq 0$ for sufficiently large values of $|\tilde{q}(t)|$, which implies that $v(t)$ is bounded from above by a non-increasing function. Since $V$ is proper $|x(t)|$ is uniformly globally bounded.

Lemma 3 The origin of the system (1) under the conditions of Theorem 4 is uniformly (asymptotically) stable. Furthermore, assume that the solutions are uniformly globally bounded; then, the origin is uniformly globally attractive.

Proof: Let the control gains be fixed according to (14) and, for any $r>0$, let the property of uniform global boundedness generate $R=\gamma(r)+c$ (see Definition 1), such that, for all $t_{\circ} \geq 0$ and all $x_{\circ} \in B_{r}, x\left(t, t_{\circ}, x_{\circ}\right) \in B_{R}$ for all $t \geq t_{\text {。 }}$. Consider a function $V: \mathbb{R}_{\geq 0} \times B_{R} \rightarrow \mathbb{R}$ defined as in (30). Under Assumption 1 its total time-derivative along the trajectories of (17), (18) satisfies, for all $(t, x) \in \mathbb{R}_{\geq 0} \times B_{R}$,

$$
\begin{aligned}
\dot{V} \leq & -\frac{\varepsilon_{1} k_{p}}{2}|\tilde{q}|^{2}-\frac{\varepsilon_{2} b d_{m}}{2}|\dot{\tilde{q}}|^{2}+\varepsilon_{1} d_{M}|\dot{\tilde{q}}|^{2} \\
& -\frac{1}{2}\left[\begin{array}{c}
|\tilde{q}| \\
|\dot{\tilde{q}}|
\end{array}\right]^{\top}\left[\begin{array}{cc}
\varepsilon_{1} k_{p} / 2 & -\varepsilon_{1} k_{c}\left(R+k_{\delta}\right) \\
* & \varepsilon_{2} b d_{m} / 2
\end{array}\right]\left[\begin{array}{c}
|\tilde{q}| \\
|\dot{\tilde{q}}|
\end{array}\right] \\
& -\frac{1}{2}\left[\begin{array}{c}
|\tilde{q}| \\
|\vartheta|
\end{array}\right]^{\top}\left[\begin{array}{cc}
\varepsilon_{1} k_{p} / 2 & -\left(\varepsilon_{1} k_{d}+\varepsilon_{2} k_{p}\right) \\
* & k_{d} a / 2 b
\end{array}\right]\left[\begin{array}{c}
|\tilde{q}| \\
|\vartheta|
\end{array}\right] \\
& -\frac{1}{2}\left[\begin{array}{l}
|\dot{\tilde{q}}| \\
|\vartheta|
\end{array}\right]^{\top}\left[\begin{array}{cc}
\frac{\varepsilon_{2} b d_{m}}{2} & -\varepsilon_{2}\left[k_{c}\left(R+k_{\delta}\right)+a d_{M}\right] \\
* & k_{d} a / 2 b
\end{array}\right]\left[\begin{array}{c}
|\dot{\tilde{q}}| \\
|\vartheta|
\end{array}\right] \\
& -\left(\frac{k_{d} a}{2 b}-\varepsilon_{2} k_{d}\right)|\vartheta|^{2}+k_{c} k_{\delta}|\dot{\tilde{q}}|^{2}
\end{aligned}
$$


where "*" stands for the opposite element in the matrix with respect to the main diagonal. Note that the second and third terms on the right-hand side of the previous inequality may be grouped together and the resulting factor of $\dot{\tilde{q}}$, i.e., $\left[\left(\varepsilon_{2} b d_{m} / 2\right)-\varepsilon_{1} d_{M}\right]$, is positive for sufficiently small values of $\varepsilon_{1} / \varepsilon_{2}$. Also, the first matrix above is positive definite if

$$
\frac{\varepsilon_{2}}{4 \varepsilon_{1}} b d_{m} \geq \frac{k_{c}^{2}\left(R+k_{\delta}\right)^{2}}{k_{p}}
$$

which holds for control gains independent of the initial conditions and of $R$, if

$$
\frac{\varepsilon_{2}}{\varepsilon_{1}}=\mathcal{O}\left(R^{2}\right)
$$

which also imposes $\varepsilon_{1} / \varepsilon_{2}$ to be "small". The second matrix is positive if

$$
\frac{\varepsilon_{1} k_{p} k_{d} a}{4 b} \geq\left(\varepsilon_{1} k_{d}+\varepsilon_{2} k_{p}\right)^{2}
$$

which holds for sufficiently small values of $\varepsilon_{1}$ and $\varepsilon_{2}$. Finally, the third matrix is positive definite if

$$
\frac{k_{d} a d_{m}}{4} \geq \varepsilon_{2}\left[\left(R+k_{\delta}\right) k_{c}+a d_{M}\right]^{2}
$$

which is satisfied for sufficiently small values of

$$
\varepsilon_{2}=\mathcal{O}\left(\frac{1}{R^{2}}\right)
$$

which in turn, in view of (34), imposes that

$$
\varepsilon_{1}=\mathcal{O}\left(\frac{1}{R^{4}}\right) \text {. }
$$

Furthermore, the factor of $\vartheta^{2}$ is negative if $\varepsilon_{2}<a / 2 b$. Note that none of these definitions violate (31) nor they restrict the gains relatively to the value of $R$. Thus, for all $(t, x) \in$ $\mathbb{R}_{\geq 0} \times B_{R}$ and for any $R \geq 0$ there exist $c^{\prime}>0$ and $\lambda \in(0,1)$ such that

$\dot{V}(t, x) \leq-c^{\prime}|x|^{2}-\frac{\lambda k_{d} a}{2 b}|\vartheta|^{2}+k_{c} k_{\delta}|\dot{\tilde{q}}|^{2} \quad \forall t \geq 0, x \in B_{R}$

with control gains independent of $R$. Therefore, recalling that $v(t):=V(t, x(t))$ we obtain

$\dot{v}(t) \leq-c^{\prime}|x(t)|^{2}-\frac{\lambda k_{d} a}{2 b}|\vartheta(t)|^{2}+k_{c} k_{\delta} R^{2} \quad \forall t_{\circ} \geq 0, x_{\circ} \in B_{r}$

On the other hand, recalling (22) we see that

$$
\dot{v}_{1}(t) \leq-\frac{\lambda k_{d} a}{2 b}|\vartheta(t)|^{2}+k_{c} k_{\delta} R^{2} \quad \forall t_{\circ} \geq 0, x_{\circ} \in B_{r} .
$$

Integrating the latter from $t_{\circ}$ to $t$, for any $t \geq t_{\circ}$, we obtain,

$$
v_{1}(t)-v_{1}\left(t_{\circ}\right) \leq-\int_{t_{\circ}}^{t}\left[\frac{\lambda k_{d} a}{2 b}|\vartheta(\tau)|^{2}-\tilde{v}\right] d \tau
$$

where $\tilde{v}:=k_{c} k_{\delta} R^{2}$. Furthermore, from (21) and the property of uniform global boundedness (see Def. 1 ), we have $v_{1}(t) \leq$ $\alpha_{1} R^{2}=\alpha_{1}[\gamma(r)+c]^{2}$ and $v_{1}\left(t_{\circ}\right) \leq \alpha_{1}\left|x_{\circ}\right|^{2}$. Therefore, defining $\beta_{r}:=[\gamma(r)+c]^{2}+r^{2}$, we obtain

$$
\int_{t_{\circ}}^{t}\left[\frac{\lambda k_{d} a}{2 b}|\vartheta(\tau)|^{2}-\tilde{v}\right] d \tau \leq \alpha_{1} \beta_{r}
$$

for any $\tilde{v}$ and for all $t \geq t_{\circ} \geq 0$.
Next, we integrate on both sides of (38) from $t_{\circ}$ to $t$, for any $t \geq t_{\circ}$ and we rearrange some terms to obtain

$c^{\prime} \int_{t_{\circ}}^{t}|x(\tau)|^{2} d \tau+\int_{t_{\circ}}^{t}\left[\frac{\lambda k_{d} a}{2 b}|\vartheta(\tau)|^{2}-\tilde{v}\right] d \tau \leq v\left(t_{\circ}\right)-v(t)$,

which implies that

$$
\begin{aligned}
c^{\prime} \int_{t_{\circ}}^{t}|x(\tau)|^{2} d \tau+\int_{t_{\circ}}^{t}\left[\frac{\lambda k_{d} a}{2 b}|\vartheta(\tau)|^{2}-\tilde{v}\right] & d \tau-\alpha_{1} \beta_{r} \\
& \leq v\left(t_{\circ}\right)-v(t) .
\end{aligned}
$$

Now, in view of (40), there exists $\varepsilon \in[0,1)$ such that

$$
c^{\prime} \int_{t_{\circ}}^{t}|x(\tau)|^{2} d \tau-\varepsilon \alpha_{1} \beta_{r} \leq v\left(t_{\circ}\right)-v(t) .
$$

Finally, reconsider (32) and let $m_{M}$ be an upper-bound on the induced norms of $M_{1}$ and $M_{2}$ then, $v(t) \leq 2 m_{M}[\gamma(r)+c]^{2}$ and $v\left(t_{\circ}\right) \leq 2 m_{M}\left|x_{\circ}\right|^{2}$. Therefore,

$$
c^{\prime} \int_{t_{\circ}}^{t}|x(\tau)|^{2} d \tau \leq\left[\alpha_{1} \varepsilon+2 m_{M}\right] \beta_{r}
$$

for all $t \geq t_{\circ}, t_{\circ} \geq 0$ and $x_{\circ} \in B_{r}$. The proof is completed by observing that the previous computations hold for arbitrary $r$ and invoking Theorem 3 with $\beta_{r \tilde{v}}:=\left(\alpha_{1} \varepsilon+2 m_{M}\right) \beta_{r}$ and $\Upsilon(s):=c^{\prime} s^{2}$.

The following statement, which is implied by Theorem 4, may be deduced from the previous proof. Yet, it improves the main result in [9] in the sense that the conditions on the control gains are significantly relaxed.

Corollary 1 The origin of the closed-loop system (1) with (15) under condition (14) is semiglobally uniformly exponentially stable.

Proof: Consider the function $V: \mathbb{R}_{\geq 0} \times \mathbb{R}^{3 n} \rightarrow \mathbb{R}_{\geq 0}$ defined in (30). Let $r$ and $R$ be two positive numbers such that $R=R(r)$. Then, following the steps of the proof of Lemma 3 to compute the total derivative of $V$, we find that, for all trajectories $x\left(t, t_{\circ}, x_{\circ}\right)$ such that $x_{\circ} \in B_{r}$ implies that $x(t) \in B_{R}$,

$$
\begin{aligned}
\dot{V} \leq-\frac{\varepsilon_{1} k_{p}|\tilde{q}|^{2}}{2} & -\left(\frac{k_{d} a}{2 b}-\varepsilon_{2} k_{d}\right)|\vartheta|^{2} \\
& -\left[\frac{\varepsilon_{2} b d_{m}}{2}-\varepsilon_{1} d_{M}-k_{c} k_{\delta}\right]|\dot{\tilde{q}}|^{2} .
\end{aligned}
$$

Therefore, it suffices to set $b \propto 1 / \varepsilon_{2}$ i.e., $b=\mathcal{O}\left(R(r)^{2}\right)$ while being sufficiently large to dominate over $k_{c} k_{\delta}$, to render the factor of $|\dot{\tilde{q}}|^{2}$ negative. Under these conditions, we conclude that $\dot{V}$ is negative definite on compacts that depend on the size of initial states and these sets may be arbitrarily enlarged for appropriate choices of the control gains.

Corollary 2 The origin of the closed-loop system (1) with (15) under condition (14) is uniformly globally asymptotically stable and exponentially stable on any compact.

We conclude that the origin is uniformly locally exponentially stable, which in turn implies that the state trajectories are uniformly square-integrable. 


\section{SySTEMS WITH RELATIVE DEGREE $m+2$}

In this section we consider the output-feedback problem, as stated in Definition 5, for systems in which case the control input enters through a chain of $m$ integrators, that is,

$$
\begin{aligned}
D(q) \ddot{q}+C(q, \dot{q}) \dot{q}+g(q) & =\xi_{1} \\
\dot{\xi}_{1} & =\xi_{2} \\
& \vdots \\
\dot{\xi}_{m} & =u
\end{aligned}
$$

where $u, \xi_{i} \in \mathbb{R}^{n}$ for all $i \leq m, m \geq 1$.

The model (42) covers several interesting cases which may be related to other challenging open problems of nonlinear control such as the control of Lagrangian (mechanical) systems, taking into account the actuator dynamics, that is,

$$
\begin{array}{r}
D(q) \ddot{q}+C(q, \dot{q}) \dot{q}+g(q)=\xi_{1} \\
\dot{x}_{a}=f_{a}\left(t, x_{a}\right)+\tau, \quad \xi_{1}=h\left(t, q, \dot{q}, x_{a}\right)
\end{array}
$$

where $x_{a}$ denotes the actuator's state and $\tau$ its control input. Provided that the actuator dynamics (43b) is input-output (globally) feedback-linearizable with respect to the output $\xi_{1}$, the model (43) may be transformed into (42). Although this task is very difficult in general, there exists a considerable bulk of literature on the subject, particularly for electrical machines -see [36]. A "simple" example concerns the flexible-joint robot manipulator model, simultaneously and independently introduced in [37], [38],

$$
\begin{aligned}
D\left(q_{1}\right) \ddot{q}_{1}+C\left(q_{1}, \dot{q}_{1}\right) \dot{q}_{1}+\mathrm{g}\left(q_{1}\right) & =K\left(q_{2}-q_{1}\right) \\
J \ddot{q}_{2}+K\left(q_{2}-q_{1}\right) & =\tau
\end{aligned}
$$

where $q_{1}$ and $q_{2}$ denote, respectively, the link and actuator generalized coordinates, $\mathrm{g}\left(q_{1}\right)$ represents potential forces, $K$ is the joint-stiffness matrix (positive diagonal), $J$ is the rotor inertia matrix and $\tau$ is the (physical) control input. It is easy to see that this system may be "transformed" into a system of the form (42) with $m=2$. For this, we define $g\left(q_{1}\right)=$ $\mathrm{g}\left(q_{1}\right)+K q_{1}, \xi_{1}=K q_{2}$ and $\xi_{2}=K \dot{q}_{2}$ then, $\dot{\xi}_{1}=\xi_{2}$ and

$$
\dot{\xi}_{2}=K J^{-1}\left[\tau-\xi_{1}+K q_{1}\right]
$$

hence, it suffices to define $\tau=\xi_{1}-K q_{1}+J K^{-1} u$. Furthermore, it is clear that the same computation goes through if (44b) contains nonlinear terms, provided that they may be canceled via output feedback, i.e., without measuring $\dot{q}_{1}$.

The solution that we propose to the output-feedback control problem for the system (42) consists in a recursive design, reminiscent of backstepping control. We design successive virtual control laws, $\xi_{i}^{*}$, which are set as references for the integrator variables $\xi_{i}$, starting with

$$
\xi_{1}^{*}=-k_{p_{0}} \tilde{q}-k_{d_{0}} \vartheta_{0}+D(q) \ddot{q}_{d}+C\left(q, \dot{q}_{d}\right) \dot{q}_{d}+g(q)
$$

$-c f .(15 \mathrm{c})$ - in the sequel, we use the symbols $k_{p_{i}}$ and $k_{d_{i}}$ to denote 'proportional' and 'derivative' control gains, defined as positive reals for all $i \in[0, m]$.
In contrast to classical backstepping control, instead of successive derivatives of $\xi_{i}^{*}$, which involve unmeasured states, we use a cascade of approximate differentiators

$$
\vartheta_{i}=\frac{b_{i} s}{s+a_{i}} \psi_{i} \quad \Leftrightarrow \quad \vartheta_{i}=\frac{b_{i}}{s+a_{i}} \dot{\psi}_{i}, \quad \forall i \in[0, m]
$$

where $\psi_{0}=\tilde{q}$ and $\psi_{i}$ is to be defined for each $i \in[1, m]$. We stress that $\vartheta_{i}$ may be defined in two equivalent manners: the first shows how the filter is to be implemented using the input $\psi_{i}$ while the second is important since it defines the dynamics of a strictly proper first order system which is internally stable, defines an output-strictly passive map $\dot{\psi}_{i} \mapsto \vartheta_{i}$ and it is input to state stable with respect to the input $\dot{\psi}_{i}$. These properties are fundamental for the analysis of the overall closed-loop system -cf. [29] and Section V.

For clarity of exposition, we first present the rationale of the control design approach for the case of a single added integrator. Then, in Section IV-B, we solve the problem for the general case, i.e., for $m>1$.

\section{A. Case of one added integrator}

We have $m=1$ hence,

$$
\dot{\xi}_{1}=u
$$

To develop intuition let us consider first the ideal control law

$$
u=-k_{p 1} \tilde{\xi}_{1}+\dot{\xi}_{1}^{*}, \quad k_{p 1}>0
$$

where $\tilde{\xi}_{1}:=\xi_{1}-\xi_{1}^{*}$. It is easy to see that in this case $\xi_{1} \rightarrow \xi_{1}^{*}$. Actually, the origin of the closed-loop equation $\dot{\tilde{\xi}}_{1}=-k_{p 1} \tilde{\xi}_{1}$ is globally exponentially stable. Furthermore, since $\xi_{1}=\tilde{\xi}_{1}+$ $\xi_{1}^{*}$ the overall closed-loop system, using (42a) and (46) with $i=0$, yields

$D(q) \ddot{\tilde{q}}+\left[C(q, \dot{q})+C\left(q, \dot{q}_{d}\right)\right] \dot{\tilde{q}}+k_{p_{0}} \tilde{q}+k_{d_{0}} \vartheta_{0}=\tilde{\xi}_{1}$

$\dot{\vartheta}_{0}=-a_{0} \vartheta_{0}+b_{0} \dot{\tilde{q}}$.

In view of Theorem 4 the origin of the system (49) with zero input (i.e., $\tilde{\xi}_{1}=0$ ) is uniformly globally asymptotically stable. Therefore, a simple cascades argument (see e.g. [39]) leads to uniform global asymptotic stability for (42) in closed loop with (45) and (48). The obvious inconvenient of (48) is that its implementation requires $\dot{\xi}_{1}^{*}$, which depends on the unmeasured velocities. Indeed ${ }^{1}$,

$$
\begin{gathered}
\dot{\xi}_{1}^{*}\left(t, q, \dot{q}, \vartheta_{0}\right)=\left[C\left(q, \dot{q}_{d}\right)+C(q, \dot{q})+C(q, \dot{q})^{\top}\right] \ddot{q}_{d}+D(q) q_{d}^{(3)} \\
+M \dot{q}_{d}+\frac{\partial g}{\partial q}{ }^{\top} \dot{q}-k_{p_{0}} \dot{\tilde{q}}-k_{d_{0}}\left[-a_{0} \vartheta_{0}+b_{0} \dot{\tilde{q}}\right]
\end{gathered}
$$

where

$$
M=\left[\begin{array}{c}
\dot{q}^{\top} \frac{\partial c_{d 1}}{\partial q}+\ddot{q}_{d}^{\top} \frac{\partial c_{d 1}}{\partial \dot{q}_{d}} \\
\vdots \\
\dot{q}^{\top} \frac{\partial c_{d n}}{\partial q}+\ddot{q}_{d}^{\top} \frac{\partial c_{d n}}{\partial \dot{q}_{d}}
\end{array}\right]
$$

and $c_{d i}$ denotes the $i$-th row of $C\left(q, \dot{q}_{d}\right)$. To overcome this difficulty, we use an approximate differentiator for $\xi_{1}^{*}$ and we

\footnotetext{
${ }^{1}$ To avoid a cumbersome notation we drop the argument $(t)$ of $q_{d}$ and its derivatives; also, we write $\dot{\tilde{q}}$ in place of $\dot{q}-\dot{q}_{d}(t)$.
} 
replace the unavailable $\dot{\xi}_{1}^{*}$ in (48) with the correction term $k_{d 1} \vartheta_{1}$, where $\vartheta_{1}$ corresponds to the dirty derivative of $\xi_{1}^{*}$, modulo an additional redesign term added to the input of the filter. That is, we modify (48) into

$$
\begin{aligned}
u & =-k_{p 1} \tilde{\xi}_{1}+k_{d 1} \vartheta_{1} \\
\vartheta_{1} & =q_{c 1}+b_{1} \xi_{1}^{*}+\zeta_{1} \\
\dot{q}_{c 1} & =-a_{1} \vartheta_{1} \\
\dot{\zeta}_{1} & =-k_{d 1} \tilde{\xi}_{1} .
\end{aligned}
$$

Note that the equations (51b)-(51c) correspond to the filter in (46), with $i=1$ and input $\dot{\psi}_{1}=\dot{\xi}_{1}^{*}+\dot{\zeta}_{1} / b_{1}$ that is, the term $\zeta_{1}$ modifies the input but not the filter dynamics. Actually, the equations (51) correspond to an implementable (outputfeedback) realization of the dynamic controller

$$
\begin{aligned}
u & =-k_{p 1} \tilde{\xi}_{1}+k_{d 1} \vartheta_{1} \\
\dot{\vartheta}_{1} & =-a_{1} \vartheta_{1}-k_{d 1} \tilde{\xi}_{1}+b_{1} \dot{\xi}_{1}^{*}
\end{aligned}
$$

with state $\vartheta_{1}$ and output $u$. Furthermore, by direct substitution of (52a) in (47), we obtain the error equation

$$
\dot{\tilde{\xi}}_{1}=-k_{p 1} \tilde{\xi}_{1}+k_{d 1} \vartheta_{1}-\dot{\xi}_{1}^{*}
$$

which is "similar" to (52b).

At this point, the motivation to include the term $-k_{d 1} \tilde{\xi}_{1}$ in equation (52b), generated via the integrator (51d), may be clearer: note that in view of the 'matching' terms $-k_{d 1} \tilde{\xi}_{1}$ and $k_{d 1} \vartheta_{1}$ the two equations (52b) and (53) form an input-to-state stable system (since $k_{p 1}, a_{1}>0$ ) with respect to the input $\dot{\xi}_{1}^{*}$. In particular, in the case that $\dot{\xi}_{1}^{*}=0$ the origin is exponentially stable. To see this, let

$$
W_{1}=\frac{1}{2}\left[\left|\tilde{\xi}_{1}\right|^{2}+\left|\vartheta_{1}\right|^{2}\right]
$$

then, a direct computation shows that its total derivative along the trajectories of (52b), (53) yields

$$
\dot{W}_{1}=-k_{p 1}\left|\tilde{\xi}_{1}\right|^{2}-a_{1}\left|\vartheta_{1}\right|^{2}+\dot{\xi}_{1}^{* \top}\left[b_{1} \vartheta_{1}-\tilde{\xi}_{1}\right]
$$

that is, $W_{1}$ is an input-to-state-stability Lyapunov function.

Thus, the system (52b), (53) is input-to-state stable with respect to the input $\dot{\xi}_{1}^{*}$ while the remaining two closed-loop equations, given by (49), form (at least locally) an input-tostate stable system from the input $\tilde{\xi}_{1}$. Actually, following the proof guidelines of Lemma 3, it may be showed that input-tostate stability holds semiglobally.

Even though these properties seem well in place for the purpose of constructing a small-gain-type argument to establish stability, the previous arguments rely on whether the reference $q_{d}$ satisfies the constraint $\dot{\xi}_{1}^{*}\left(t, q_{d}, \dot{q}_{d}, 0\right) \equiv 0$. This is necessary for the origin (in terms of a closed-loop system's state, including the tracking errors) to be an equilibrium point. In order to lift this restriction on $q_{d}$, which may appear conservative in some cases, we further modify the controller (51) by introducing some feedforward terms. That is, let

$$
\begin{aligned}
u & =-k_{p 1} \tilde{\xi}_{1}+k_{d 1} \vartheta_{1}+\dot{\xi}_{1}^{*}\left(t, q_{d}, \dot{q}_{d}, 0\right) \\
\vartheta_{1} & =q_{c 1}+b_{1} \xi_{1}^{*}+\zeta_{1} \\
\dot{q}_{c 1} & =-a_{1} \vartheta_{1} \\
\dot{\zeta}_{1} & =-k_{d 1} \tilde{\xi}_{1}-b_{1} \dot{\xi}_{1}^{*}\left(t, q_{d}, \dot{q}_{d}, 0\right)
\end{aligned}
$$

and, to shorten the notation, define $\dot{\xi}_{1 \circ}^{*}:=\dot{\xi}_{1}^{*}\left(t, q_{d}, \dot{q}_{d}, 0\right)$. A direct computation shows that the closed-loop equations now correspond to (49) and, instead of (52b) and (53), we have

$$
\begin{aligned}
& \dot{\tilde{\xi}}_{1}=-k_{p 1} \tilde{\xi}_{1}+k_{d 1} \vartheta_{1}-\left[\dot{\xi}_{1}^{*}-\dot{\xi}_{1 \circ}^{*}\right] \\
& \dot{\vartheta}_{1}=-a_{1} \vartheta_{1}-k_{d 1} \tilde{\xi}_{1}+b_{1}\left[\dot{\xi}_{1}^{*}-\dot{\xi}_{1 \circ}^{*}\right] .
\end{aligned}
$$

Note that the introduction of the feedforward term $\dot{\xi}_{1 \circ}^{*}$ only shifts the equilibrium of the closed-loop system, leaving the internal controller structure unchanged. Therefore, the previous claims on internal stability (without input) as well as inputoutput and input-to-state stability continue to hold, only with respect to a different input. In particular, the interconnected systems (56) form an input-to-state stable system with respect to the input $\left[\dot{\xi}_{1}^{*}-\dot{\xi}_{1 \circ}^{*}\right]$ and with Lyapunov function $W_{1}$. The derivative of the latter, along the trajectories of (56), yields

$$
\dot{W}_{1}=-k_{p 1}\left|\tilde{\xi}_{1}\right|^{2}-a_{1}\left|\vartheta_{1}\right|^{2}+\left[\dot{\xi}_{1}^{*}-\dot{\xi}_{1 \circ}^{*}\right]^{\top}\left[b_{1} \vartheta_{1}-\tilde{\xi}_{1}\right] .
$$

Thus, with aim at developing a small-gain type argument, we need to establish input-to-state stability with respect to the tracking errors. To that end, we introduce a (mild) additional technical hypothesis on the system (42). Assumption 2, below, together with (11) and Assumption 1, guarantees that $\dot{\xi}_{1}^{*}\left(t, q, \dot{q}, \vartheta_{0}\right)$ is globally Lipschitz in the last two arguments, uniformly in $t$, and that it is bounded in the first two arguments. More precisely, there exist non-negative real numbers $\eta_{1}, \eta_{2}$ and $\eta_{3}$, as well as a continuous saturation function sat $: \mathbb{R} \rightarrow \mathbb{R}$ such that $y \operatorname{sat}(y)>0$ for all $y \neq 0$ and $|\operatorname{sat}(y)| \leq 1$, such that,

$$
\left|\left[\dot{\xi}_{1}^{*}-\dot{\xi}_{1 \circ}^{*}\right]\right| \leq \eta_{1} \operatorname{sat}(|\tilde{q}|)+\eta_{2}|\dot{\tilde{q}}|+\eta_{3}\left|\vartheta_{0}\right| .
$$

Assumption 2 (1) let the Coriolis and centrifugal forces function $(w, y) \mapsto C$ be globally Lipschitz and uniformly bounded in $w$, i.e., assume that there exists a saturation function sat, as defined above, such that, for all $w, y, z \in \mathbb{R}^{n}$,

$$
|C(w, y)-C(z, y)| \leq \operatorname{sat}(|w-z|)|y|
$$

(2) let the function representing the potential-energy force satisfy

$$
\exists k_{v}>0: \quad\left|\frac{\partial g}{\partial q}\right| \leq k_{v}, \quad \forall q \in \mathbb{R}^{n} .
$$

Remark 4 Assumption 2 is fairly weak; it holds, e.g., for robot manipulators with only prismatic or only revolute joints, since $w \mapsto C$ and $q \mapsto g$ are defined via constants and trigonometric or linear-growth functions. Readers interested in the regularity properties of the Coriolis matrix are invited to see [32]. On the other hand, the Jacobian of $g(q)$ is thoroughly studied in [40]. For the particular case of flexible-joint robots (44), we have $g\left(q_{1 d}\right)=\mathrm{g}\left(q_{1 d}\right)+K q_{1 d}$ hence, inequality (59) holds if $\left|\frac{\partial \mathrm{g}}{\partial q}\right| \leq k_{v}$ and $K$ is bounded

We are now ready to present the main result of this section.

Theorem 5 Consider the system (49), (50), (56), which corresponds to the closed-loop of (42), (45) and (55). Let $q_{d}$ be given as in Definition 5, let Assumptions 1 and 2 hold and the 
control gains satisfy: $k_{p_{0}}>1$,

$$
\begin{gathered}
k_{d_{0}\left[\frac{a_{0}}{4 b_{0}}-1\right]}>\frac{3}{2}+\frac{\left(2 k_{c} k_{\delta}+3 / 2\right) a_{0}^{2}}{b_{0}^{2}} \\
k_{p 1}>\frac{1}{2}\left(\eta_{2}^{2}+\eta_{3}^{2}\right)+2 \\
a_{1}>\frac{1}{2}\left(\eta_{2}^{2}+\eta_{3}^{2}\right) b_{1}^{2}+1 .
\end{gathered}
$$

Then, the origin is uniformly globally asymptotically stable.

Proof: The state of the closed-loop system, which is given by Equations (49) and (56), is denoted by

$$
x=\left[\begin{array}{lllll}
\tilde{q}^{\top} & \dot{\tilde{q}}^{\top} & \vartheta_{0}^{\top} & \tilde{\xi}_{1}^{\top} & \vartheta_{1}^{\top}
\end{array}\right]^{\top} .
$$

We establish uniform global asymptotic stability of $\{x=0\}$ by showing uniform forward completeness, uniform global boundedness and uniform global attractivity.

Uniform forward completeness. Consider the function $V_{1}$ as defined in (20), for the system (49) that is,

$V_{1}\left(t, \tilde{q}, \dot{\tilde{q}}, \vartheta_{0}\right)=\frac{1}{2}\left(\dot{\tilde{q}}^{\top} D\left(\tilde{q}+q_{d}(t)\right) \dot{\tilde{q}}+k_{p_{0}}|\tilde{q}|^{2}+\frac{k_{d_{0}}}{b_{0}}\left|\vartheta_{0}\right|^{2}\right)$.

The total derivative of $\mathcal{V}_{1}:=V_{1}+W_{1}$ along the trajectories of (49), (56) yields

$$
\begin{aligned}
\dot{\mathcal{V}}_{1}= & -\frac{a_{0} k_{d_{0}}}{b_{0}}\left|\vartheta_{0}\right|^{2}+k_{c} k_{\delta}|\dot{\tilde{q}}|^{2}+\dot{\tilde{q}}^{\top} \tilde{\xi}_{1} \\
& -k_{p 1}\left|\tilde{\xi}_{1}\right|^{2}-a_{1}\left|\vartheta_{1}\right|^{2}+\left[\dot{\xi}_{1}^{*}-\dot{\xi}_{1 \mathrm{o}}^{*}\right]^{\top}\left[b_{1} \vartheta_{1}-\tilde{\xi}_{1}\right]
\end{aligned}
$$

where $\dot{\xi}_{1}^{*}$ is given in (50). On the other hand, the term $\left[\dot{\xi}_{1}^{*}-\right.$ $\left.\dot{\xi}_{1 \circ}^{*}\right]$ satisfies, in view of Assumption 2, the "Lipschitz bound" (58). Using this and the triangle inequality, it follows that the last term on the right-hand side of (63), which comes from (57), satisfies

$$
\begin{gathered}
{\left[\dot{\xi}_{1}^{*}-\dot{\xi}_{10}^{*}\right]^{\top}\left[b_{1} \vartheta_{1}-\tilde{\xi}_{1}\right] \leq|\dot{\tilde{q}}|^{2}+\left|\vartheta_{0}\right|^{2}+\eta_{1} \operatorname{sat}(|\tilde{q}|)\left[b_{1}\left|\vartheta_{1}\right|+\left|\tilde{\xi}_{1}\right|\right]} \\
+\frac{\left(\eta_{2}^{2}+\eta_{3}^{2}\right)}{2}\left[b_{1}^{2}\left|\vartheta_{1}\right|^{2}+\left|\tilde{\xi}_{1}\right|^{2}\right] .
\end{gathered}
$$

Using the latter in (63) and the triangle inequality on $\dot{\tilde{q}}^{\top} \tilde{\xi}_{1}$, we get

$$
\begin{aligned}
\dot{\mathcal{V}}_{1} \leq & -\left[\frac{a_{0} k_{d_{0}}}{b_{0}}-1\right]\left|\vartheta_{0}\right|^{2}+\left[k_{c} k_{\delta}+\frac{3}{2}\right]|\dot{\tilde{q}}|^{2} \\
& -\left[a_{1}-\frac{b_{1}^{2}}{2}\left(\eta_{2}^{2}+\eta_{3}^{2}\right)\right]\left|\vartheta_{1}\right|^{2}+\eta_{1} \operatorname{sat}(|\tilde{q}|)\left[b_{1}\left|\vartheta_{1}\right|+\left|\tilde{\xi}_{1}\right|\right] . \\
& -\left[k_{p 1}-\frac{1}{2}\left(1+\eta_{2}^{2}+\eta_{3}^{2}\right)\right]\left|\tilde{\xi}_{1}\right|^{2}
\end{aligned}
$$

It follows that there exist $c$ and $c^{\prime}>0$ such that $\dot{\mathcal{V}}_{1} \leq c \mathcal{V}_{1}+$ $c^{\prime}$ hence, integrating the latter and invoking the comparison principle, as in the proof of Lemma $1-c f$. (22), we obtain that the closed-loop system is uniformly forward complete that is, the closed-loop trajectories satisfy (19) with an appropriate redefinition of the state and of the constants $c_{1}$ and $c_{2}$.

Uniform global boundedness. We proceed by contradiction, based on the proof of Lemma 2. Let $|x(t)| \rightarrow \infty$ as $t \rightarrow \infty$ and consider two cases: first, assume that $\tilde{\xi}_{1}$ and $\vartheta_{1}$ are uniformly bounded then, in view of (65), there exists $c>0$ such that

$$
\dot{\mathcal{V}}_{1} \leq-\left[\frac{a_{0} k_{d_{0}}}{b_{0}}-1\right]\left|\vartheta_{0}\right|^{2}+\left[k_{c} k_{\delta}+\frac{3}{2}\right]|\dot{\tilde{q}}|^{2}+c .
$$

If on the contrary, $\left|\tilde{\xi}_{1}(t) \vartheta_{1}(t)\right| \rightarrow \infty$ as $t \rightarrow \infty$ then $^{2}$, for sufficiently large $t$ the sum of the last three terms on the righthand side of (65) becomes non-positive so (66) holds with $c=0$ for large $t$. In either case, the rest of the proof follows as in Lemma $2-c f$. (22).

Uniform global attractivity. We proceed as in the proof of Lemma 3. In view of uniform global boundedness for each $r>0$ there exists $R(r)$ such that if $x\left(t_{\circ}\right) \in B_{r}$ then $x(t) \in$ $B_{R}$ for all $t \geq t_{\circ}$ where $x$ is defined in (61).

Consider the function $\mathcal{V}: \mathbb{R}_{\geq 0} \times B_{R} \rightarrow \mathbb{R}$ defined by

$$
\mathcal{V}(t, x)=V_{1}\left(t, \tilde{q}, \dot{\tilde{q}}, \vartheta_{0}\right)+V_{2}\left(t, \tilde{q}, \dot{\tilde{q}}, \vartheta_{0}\right)+W_{1}\left(\tilde{\xi}_{1}, \vartheta_{1}\right)
$$

where $V_{1}$ and $V_{2}$ are defined as in (62) and (26) respectively. With some obvious modifications, in view of Assumptions 1 and 2, the condition ${ }^{3}$ (14), and after the proof of Lemma 3, we obtain

$$
\begin{aligned}
\dot{V}_{1}+\dot{V}_{2} \leq & -\frac{1}{2}\left[\varepsilon_{1} k_{p_{0}}|\tilde{q}|^{2}+\varepsilon_{2} b_{0} d_{m}|\dot{\tilde{q}}|^{2}\right] \\
& +\left(k_{c} k_{\delta}+\varepsilon_{1} d_{M}\right)|\dot{\tilde{q}}|^{2}-\left[\frac{k_{d_{0}} a_{0}}{2 b_{0}}-\varepsilon_{2} k_{d_{0}}\right]\left|\vartheta_{0}\right|^{2} \\
& +\tilde{\xi}_{1}^{\top}\left[\dot{\tilde{q}}+\varepsilon_{1} \tilde{q}-\varepsilon_{2} \vartheta_{0}\right]
\end{aligned}
$$

$-c f$. (33). Applying the triangle inequality to the last term on the right-hand side of the previous inequality we obtain

$$
\begin{aligned}
\dot{V}_{1}+\dot{V}_{2} \leq & \left(k_{c} k_{\delta}+\varepsilon_{1} d_{M}+\frac{1}{2}\right)|\dot{\tilde{q}}|^{2}+\frac{1}{2}\left(1+\varepsilon_{1}+\varepsilon_{2}\right)\left|\tilde{\xi}_{1}\right|^{2} \\
& -\frac{1}{2}\left[\varepsilon_{1}\left(k_{p_{0}}-1\right)|\tilde{q}|^{2}+\varepsilon_{2} b_{0} d_{m}|\dot{\tilde{q}}|^{2}\right] \\
& -\left[\frac{k_{d_{0}} a_{0}}{2 b_{0}}-\varepsilon_{2}\left(k_{d_{0}}+\frac{1}{2}\right)\right]\left|\vartheta_{0}\right|^{2}
\end{aligned}
$$

Next, define

$$
\begin{aligned}
\alpha_{1} & =\frac{k_{d_{0}} a_{0}}{2 b_{0}}-\varepsilon_{2}\left(k_{d_{0}}+\frac{1}{2}\right)-1 \\
\alpha_{2} & =k_{p 1}-\frac{1}{2}\left(\eta_{2}^{2}+\eta_{3}^{2}+2+\varepsilon_{1}+\varepsilon_{2}\right) \\
\alpha_{3} & =a_{1}-\frac{1}{2}\left(\eta_{2}^{2}+\eta_{3}^{2}\right) b_{1}^{2}-1 \\
\alpha_{4} & =k_{c} k_{\delta}+\frac{3}{2}+\varepsilon_{1} d_{M}
\end{aligned}
$$

and, for each $\mu>0, \alpha_{5}(\mu)=\eta_{1}^{2}\left(b_{1}^{2}+1\right) \operatorname{sat}(\mu)^{2} / 2$. Note that $\alpha_{1}, \alpha_{2}$ and $\alpha_{3}$ are positive in view of (60a). Then, adding (67) to (57) and using (64) we finally obtain

$$
\begin{aligned}
\dot{\mathcal{V}}(t, x) \leq-\frac{1}{2}[ & \left.\varepsilon_{1}\left(k_{p_{0}}-1\right)|\tilde{q}|^{2}+\varepsilon_{2} b_{0} d_{m}|\dot{\tilde{q}}|^{2}\right]-\alpha_{1}\left|\vartheta_{0}\right|^{2} \\
& -\alpha_{2}\left|\tilde{\xi}_{1}\right|^{2}-\alpha_{3}\left|\vartheta_{1}\right|^{2}+\alpha_{4}|\dot{\tilde{q}}|^{2}+\alpha_{5}(|\tilde{q}|) .
\end{aligned}
$$

Next, let uniform global boundedness generate $R=\gamma(r)+c$ such that $\max \{|\tilde{q}(t)|,|\dot{\tilde{q}}(t)|\} \leq R$ for all $t \geq t_{\circ} \geq 0$, and define $z^{\top}=\left[\begin{array}{llll}\tilde{q}^{\top} & \dot{\tilde{q}}^{\top} & \tilde{\xi}_{1}^{\top} & \vartheta_{1}^{\top}\end{array}\right]$,

$$
c^{\prime}=\min \left\{\varepsilon_{1}\left(k_{p_{0}}-1\right), \varepsilon_{2} b_{0} d_{m}, \alpha_{2}, \alpha_{3}\right\} .
$$

We have, for all $t \geq t_{\circ} \geq 0$, all $r>0$ and all $x_{\circ} \in B_{r}$,

$$
\dot{\mathcal{V}}(t, x(t)) \leq-c^{\prime}|z(t)|^{2}-\alpha_{1}\left|\vartheta_{0}(t)\right|^{2}+\left[\alpha_{5}(R)+\alpha_{4} R^{2}\right]
$$

which is similar to (38). The rest of the proof follows as the proof of Lemma 3, by invoking Theorem 3.

\footnotetext{
${ }^{2}$ In view of uniform forward completeness, the solutions may either be uniformly bounded or grow unboundedly, also uniformly.

${ }^{3}$ Note that (60a) implies (14) hence we may proceed as in the proof of Lemma 3.
} 


\section{B. Case of $m>1$ added integrators}

Based on the previous developments, we follow a controldesign approach reminiscent of backstepping in which approximate differentiators are used at each step in place of the unavailable derivatives of intermediate virtual control inputs, $\dot{\xi}_{i}^{*}$. To start with, let the reference to the second integrator, i.e., $\xi_{2}^{*}$, correspond to the right-hand side of (55a) and let the subsequent references, $\xi_{j>2}^{*}$, be defined in a similar manner, that is,

$$
\left.\begin{array}{rl}
\xi_{i+1}^{*} & =-k_{p_{i}} \tilde{\xi}_{i}+k_{d_{i}} \vartheta_{i}+\dot{\xi}_{i \circ}^{*} \quad \forall i \in[1, m-1] \\
\vartheta_{i} & =q_{c i}+b_{i} \xi_{i}^{*}+\zeta_{i} \\
\dot{q}_{c i} & =-a_{i} \vartheta_{i} \\
\dot{\zeta}_{i} & =-\left(k_{d_{i}}-\sigma_{i}\right) \tilde{\xi}_{i}-b_{i} \dot{\xi}_{i \circ}^{*}
\end{array}\right\} \quad \forall i \in[1, m]
$$

where $\sigma_{i}$ is a control "redesign" constant gain defined as

$$
\sigma_{1}=0, \quad \sigma_{i}=b_{i} k_{p_{i-1}} \forall i \in[2, m]
$$

and $\dot{\xi}_{i \circ}^{*}$ is a feedforward term that is function of $t$ and is defined as the $i$ th derivative of $\xi_{10}^{*}$ which, according to (45), corresponds to $\xi_{10}^{*}:=D\left(q_{d}\right) \ddot{q}_{d}+C\left(q_{d}, \dot{q}_{d}\right) \dot{q}_{d}+g\left(q_{d}\right)$. The purpose of the feedforward term $\dot{\xi}_{i \circ}^{*}$ is, as in (55), to place the equilibrium of the closed-loop system at the origin without restricting the desired reference trajectories $q_{d}$. The choice of the notation " $\dot{\xi}_{i \circ}^{*}$ " comes from the fact that $\dot{\xi}_{i \circ}^{*}$ corresponds to the steady-state value of the virtual control law $\xi_{i+1}^{*}$ at the origin $\left(x, \tilde{\xi}_{i}, \vartheta_{i}\right)=(0,0,0)$ hence, we recover $\xi_{i+1}^{*}=\dot{\xi}_{i \circ}^{*}$ which may be viewed as a reference dynamics for each of the system's equations $\dot{\xi}_{i}=\xi_{i+1}$.

Let us proceed to write the error dynamics equations. To that end, let $\tilde{\xi}_{i}=\xi_{i}-\xi_{i}^{*}$ then, the equation $\dot{\xi}_{i}=\xi_{i+1}$ is equivalent to $\dot{\tilde{\xi}}_{i}=\tilde{\xi}_{i+1}+\xi_{i+1}^{*}-\dot{\xi}_{i}^{*}$ and, from (68), we have, for all $i \in[1, m]$,

$$
\begin{aligned}
& \dot{\tilde{\xi}}_{i}=-k_{p_{i}} \tilde{\xi}_{i}+k_{d_{i}} \vartheta_{i}-\left[\dot{\xi}_{i}^{*}-\dot{\xi}_{i \circ}^{*}\right]+\tilde{\xi}_{i+1} \\
& \dot{\vartheta}_{i}=-a_{i} \vartheta_{i}-\left(k_{d_{i}}-\sigma_{i}\right) \tilde{\xi}_{i}+b_{i}\left[\dot{\xi}_{i}^{*}-\dot{\xi}_{i o}^{*}\right]
\end{aligned}
$$

with $\tilde{\xi}_{m+1}:=0$. Reminiscent of the case $m=1$, for each $i>$ 1 , these equations form a pair of interconnected stable linear systems with input $\left[\dot{\xi}_{i}^{*}-\dot{\xi}_{i \circ}^{*}\right]$. For the sake of analyzing the stability based on a small-gain-based reasoning we show that Equations (69) may be expressed in the form of interconnected stable linear multivariable systems with "input" $\left[\dot{\xi}_{1}^{*}-\dot{\xi}_{10}^{*}\right]$. See the shadowed block labeled $\Sigma_{i}$ in Figure 1.

To that end, we first develop each element of $\dot{\xi}^{*}=$ $\left[\dot{\xi}_{1}^{*} \cdots \dot{\xi}_{m}^{*}\right]^{\top}$ as a function of $\tilde{\xi}=\left[\tilde{\xi}_{1} \cdots \tilde{\xi}_{m}\right]^{\top}, \vartheta=$ $\left[\vartheta_{1} \cdots \vartheta_{m}\right]^{\top}$ and $\dot{\xi}_{1}^{*}$. In view of (68a) and (69) we have, for all $i \in[2, m]$,

$$
\begin{aligned}
\dot{\xi}_{i}^{*}=[ & \left.k_{p_{i-1}}^{2}-k_{d_{i-1}}^{2}+k_{d_{i-1}} \sigma_{i-1}\right] \tilde{\xi}_{i-1} \\
& -k_{d_{i-1}}\left[k_{p_{i-1}}+a_{i-1}\right] \vartheta_{i-1}-k_{p_{i-1}} \tilde{\xi}_{i} \\
& +\left[k_{p_{i-1}}+k_{d_{i-1}} b_{i-1}\right]\left[\dot{\xi}_{i-1}^{*}-\dot{\xi}_{i-10}^{*}\right]+\ddot{\xi}_{i-10}^{*},
\end{aligned}
$$

which exhibits the recursive definition of $\dot{\xi}_{i}^{*}$. Figure 1 illustrates the fact that the recursive definition of these references leads to nested feedback interconnections of input-to-state stable systems. In view of this recursiveness and of the linearity of $\dot{\xi}_{i}^{*}$ as a function of $\tilde{\xi}_{i-1}$ and $\vartheta_{i-1}$, a direct albeit long computation -see the Appendix in [34], shows that for all $i \in[2, m]$,

$\dot{\xi}_{i}^{*}=-k_{p_{i-1}} \tilde{\xi}_{i}+\sum_{k=1}^{i-1} \eta_{i_{k}} \tilde{\xi}_{k}-\mu_{i_{k}} \vartheta_{k}+\prod_{j=1}^{i-1} \beta_{j}\left[\dot{\xi}_{1}^{*}-\dot{\xi}_{1 \mathrm{o}}^{*}\right]+\dot{\xi}_{i \circ}^{*}$

where

$$
\beta_{j}=k_{p_{j}}+b_{j} k_{d_{j}}, \quad j \in[1, m-1]
$$

and, for each ${ }^{4} i \in[2, m]$ and $k \in[1, i-1]$,

$$
\begin{gathered}
\eta_{i_{k}}=\prod_{j=k+1}^{i} \beta_{j}^{\operatorname{sgn}(i-j)}\left[k_{p_{k}}^{2}-k_{d_{k}}^{2}-k_{p_{k}} k_{p_{k-1}} \operatorname{sgn}(k-1)\right], \\
\mu_{i_{k}}=\prod_{j=k+1}^{i} \beta_{j}^{\operatorname{sgn}(i-j)} k_{d_{k}}\left[k_{p_{k}}+a_{k}\right] .
\end{gathered}
$$

Therefore, collecting Equations (70) in vector form, we obtain

$$
\dot{\xi}^{*}=\Gamma_{1} \tilde{\xi}+\Gamma_{2} \vartheta+\Gamma_{3}\left[\dot{\xi}_{1}^{*}-\dot{\xi}_{10}^{*}\right]+\dot{\xi}_{\circ}^{*}
$$

with

$$
\begin{gathered}
\Gamma_{1}=\left[\begin{array}{cccc}
0 & 0 & \cdots & 0 \\
\eta_{2_{1}} & -k_{p_{1}} & & \vdots \\
\vdots & & \ddots & \\
\eta_{m_{1}} & \eta_{m_{2}} & \cdots & -k_{p_{m-1}}
\end{array}\right] \\
\Gamma_{2}=\left[\begin{array}{cccc}
0 & 0 & \cdots & 0 \\
-\mu_{2_{1}} & 0 & & \vdots \\
\vdots & & \ddots & \\
-\mu_{m_{1}} & -\mu_{m_{2}} & \cdots & 0
\end{array}\right] . \quad \Gamma_{3}=\left[\begin{array}{c}
1 \\
\beta_{1} \\
\beta_{1} \beta_{2} \\
\vdots
\end{array}\right] .
\end{gathered}
$$

Furthermore, we also "collect" the control gains $a_{i}, b_{i}, k_{d_{i}}, k_{p_{i}}$ etc., into the diagonal matrices $A:=\operatorname{diag}\left\{a_{i}\right\}, B:=\operatorname{diag}\left\{b_{i}\right\}$, $K_{d}:=\operatorname{diag}\left\{k_{d_{i}}\right\}, K_{d}^{\prime}:=K_{d}-\operatorname{diag}\left\{\sigma_{i}\right\}$, and we define

$$
\begin{aligned}
\tilde{\xi}^{\top} & =\left[\begin{array}{lll}
\tilde{\xi}_{1}^{\top} & \cdots & \tilde{\xi}_{m}^{\top}
\end{array}\right], \quad \vartheta^{\top}=\left[\begin{array}{lll}
\vartheta_{1}^{\top} & \cdots & \vartheta_{m}^{\top}
\end{array}\right], \quad K_{p}=\left[\begin{array}{ccccc}
k_{p_{1}} & -1 & 0 & \cdots & 0 \\
0 & \ddots & \ddots & & \vdots \\
\vdots & & & & -1 \\
0 & \cdots & \cdots & 0 & k_{p_{m}}
\end{array}\right] . \\
\dot{\xi}_{\circ}^{* \top} & =\left[\begin{array}{llll}
\dot{\xi}_{1 \circ}^{* \top} & \cdots & \dot{\xi}_{m \circ}^{* \top}
\end{array}\right],
\end{aligned}
$$

Hence, Equations (69) become

$$
\begin{aligned}
& \dot{\tilde{\xi}}=-K_{p} \tilde{\xi}+K_{d} \vartheta-\left[\dot{\xi}^{*}-\dot{\xi}_{\circ}^{*}\right] \\
& \dot{\vartheta}=-A \vartheta-K_{d}^{\prime} \tilde{\xi}+B\left[\dot{\xi}^{*}-\dot{\xi}_{\circ}^{*}\right] .
\end{aligned}
$$

-cf. (56). Using (74), Equations (75) take the desirable form

$$
\begin{aligned}
& \dot{\tilde{\xi}}=-\left(K_{p}+\Gamma_{1}\right) \tilde{\xi}+\left(K_{d}-\Gamma_{2}\right) \vartheta-\Gamma_{3}\left[\dot{\xi}_{1}^{*}-\dot{\xi}_{1 \mathrm{o}}^{*}\right] \\
& \dot{\vartheta}=-\left(A-B \Gamma_{2}\right) \vartheta-\left(K_{d}^{\prime}-B \Gamma_{1}\right) \tilde{\xi}+B \Gamma_{3}\left[\dot{\xi}_{1}^{*}-\dot{\xi}_{1 \mathrm{o}}^{*}\right]
\end{aligned}
$$

which consists in a linear multivariable autonomous system with input $\left[\dot{\xi}_{1}^{*}-\dot{\xi}_{10}^{*}\right]$, which vanishes at $\left(\tilde{q}, \dot{\tilde{q}}, \vartheta_{0}\right)=(0,0,0)$.

Thus, provided that one choose $A, K_{p}, K_{d}$ and $B$ to render the origin of (76), subject to $\dot{\xi}_{1}^{*}=\dot{\xi}_{1 \circ}^{*}$, globally exponentially stable, we obtain input-to-state stability of (76) with respect to the input $\left[\dot{\xi}_{1}^{*}-\dot{\xi}_{1 \mathrm{o}}^{*}\right]$. Moreover, we recall that $\dot{\xi}_{1}^{*}\left(t, q, \dot{q}, \vartheta_{0}\right)$

\footnotetext{
${ }^{4}$ With an abuse of notation, in (72) and (73) we consider that $\operatorname{sgn}(0)=0$.
} 


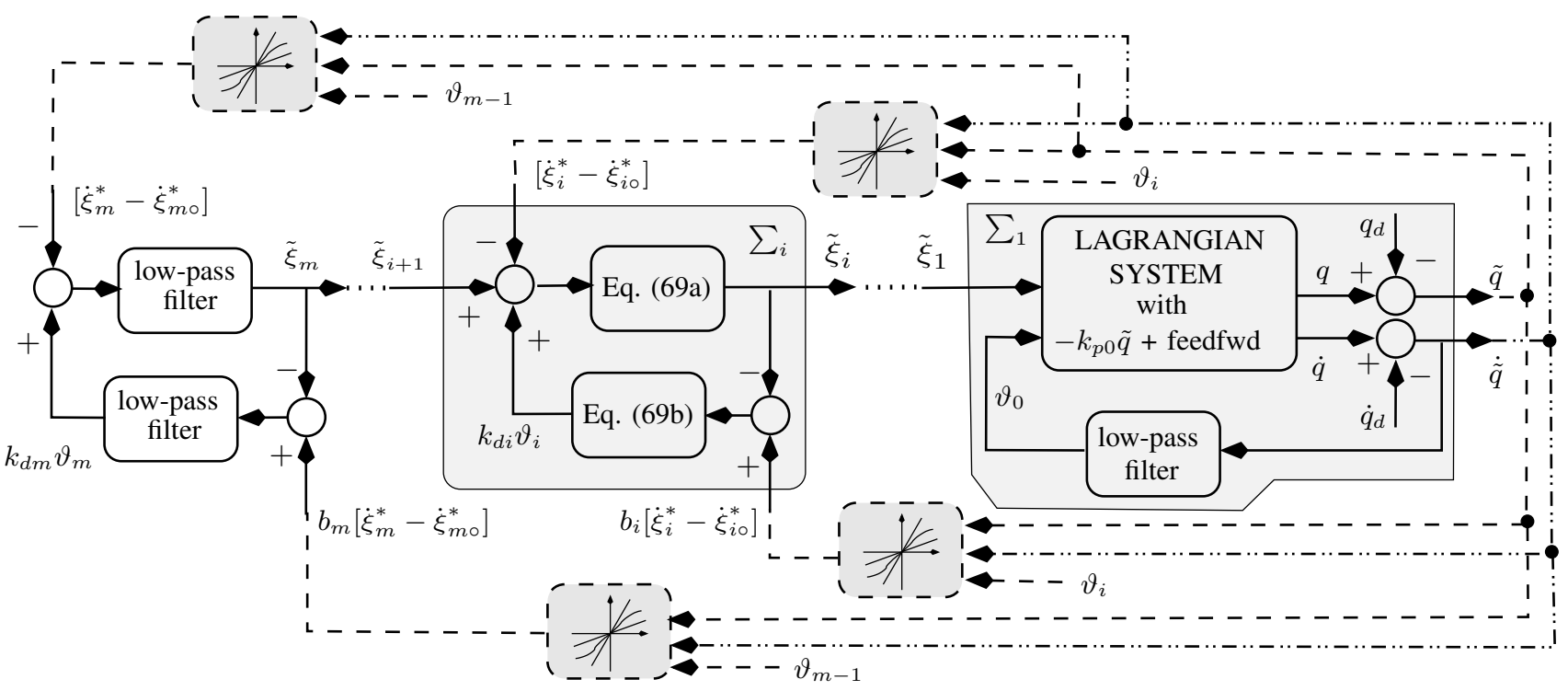

Fig. 1. Schematic block-diagram of the error dynamics, not the control implementation. The closed-loop system consists in a series of nested feedbackinterconnections of input-output, input-to-state stable systems.

is globally Lipschitz in $q, \dot{q}$ and $\vartheta_{0}$, uniformly in $t$ therefore, (76) is input-to-state stable with respect to the input $\left(\tilde{q}, \dot{\tilde{q}}, \vartheta_{0}\right)$. On the other hand, the rest of the closed-loop equations, i.e., (49), form (at least locally) an input-to-state stable system with respect to the input $\tilde{\xi}_{1}$. The following statement on uniform global asymptotic stability relies on these observations and an inductive trajectory-based analysis along similar proof-lines as those of Theorem 5 .

Remark 5 The controller (68) relies on the measurement of $\xi_{i}$ for all $i \in[1, m]$ however, a certainty-equivalence controller with a Luenberger observer for $\xi$ may be used, based on the measurement of $\xi_{1}$ only. This is not developed here.

Theorem 6 Let $q_{d}$ be given as in Definition 5. Consider the system (42) and the output-feedback dynamic controller (45), (68) under Assumptions 1 and 2. Consider $\mathcal{A}$ and $\mathcal{B}$ as defined on top of next page. Assume that the control gains are such that $\mathcal{A}$ is Hurwitz,

$$
k_{d_{0}}\left[\frac{a_{0}}{4 b_{0}}-m\right]>\frac{m+2}{2}+\frac{\left[2 k_{c} k_{\delta}+(m+2) / 2\right] a_{0}^{2}}{b_{0}^{2}}
$$

and there exist positive definite matrices $Q$ and $P$ such that $Q=-\mathcal{A}^{\top} P-P \mathcal{A}$ and

$$
Q>\left(\eta_{2}^{2}+\eta_{3}^{2}\right) \operatorname{diag}\left\{\left|[P \mathcal{B}]_{i}\right|^{2}\right\}
$$

where $[P \mathcal{B}]_{i}$ with $i \in\{1 \ldots 2 m\}$ denotes the ith $n \times n$ block of $P \mathcal{B} \in \mathbb{R}^{2 m n \times n}$. Then, the origin of the closed-loop system is uniformly globally asymptotically stable.

Sketch of proof. The proof follows mutatis mutandis that of Theorem 5. Defining $x:=\left[\begin{array}{ll}\tilde{\xi} & \vartheta\end{array}\right]^{\top}$, the equations (76) become

$$
\dot{x}=\mathcal{A} x+\mathcal{B}\left[\dot{\xi}_{1}^{*}-\dot{\xi}_{10}^{*}\right]
$$

and the total derivative of

$$
W(x)=\frac{1}{2} x^{\top} P x
$$

along the trajectories generated by (79) satisfies

$$
\dot{W} \leq-\frac{1}{2} x^{\top} Q x+x^{\top} P \mathcal{B}\left[\dot{\xi}_{1}^{*}-\dot{\xi}_{1 \mathrm{o}}^{*}\right] .
$$

On the other hand, using (58) we obtain

$$
\begin{array}{r}
x^{\top} P \mathcal{B}\left[\dot{\xi}_{1}^{*}-\dot{\xi}_{1 \circ}^{*}\right]=\left(\sum_{i=1}^{m} \tilde{\xi}_{i}^{\top}[P \mathcal{B}]_{i}+\vartheta_{i}^{\top}[P \mathcal{B}]_{m+i}\right)\left[\dot{\xi}_{1}^{*}-\dot{\xi}_{1 \circ}^{*}\right] \\
\leq \frac{1}{2}\left(\sum_{i=1}^{m}\left|\tilde{\xi}_{i}\right|^{2}\left|[P \mathcal{B}]_{i}\right|^{2}+\left|\vartheta_{i}\right|^{2}\left|[P \mathcal{B}]_{m+i}\right|^{2}\right)\left(\eta_{2}^{2}+\eta_{3}^{2}\right) \\
+\eta_{1}\left(\sum_{i=1}^{m}\left|\tilde{\xi}_{i}\right|\left|[P \mathcal{B}]_{i}\right|+\left|\vartheta_{i}\right|\left|[P \mathcal{B}]_{m+i}\right|\right)+\frac{m}{2}\left[|\dot{\tilde{q}}|^{2}+\left|\vartheta_{0}^{2}\right|\right] .
\end{array}
$$

hence the total derivative of

$$
\mathcal{V}=\frac{1}{2}\left(\dot{\tilde{q}}^{\top} D\left(\tilde{q}+q_{d}(t)\right) \dot{\tilde{q}}+k_{p_{0}}|\tilde{q}|^{2}+\frac{k_{d_{0}}}{b_{0}}\left|\vartheta_{0}\right|^{2}\right)+W(x)
$$

yields

$$
\begin{aligned}
\dot{\mathcal{V}} \leq-\left[\frac{a_{0} k_{d_{0}}}{b_{0}}-\frac{m}{2}\right]\left|\vartheta_{0}\right|^{2}+\left[k_{c} k_{\delta}+\frac{m+2}{2}\right]|\dot{\tilde{q}}|^{2} & \\
-\frac{1}{2} x^{\top} & {\left[Q-\left(\eta_{2}^{2}+\eta_{3}^{2}\right) \operatorname{diag}\left\{\left|[P \mathcal{B}]_{i}\right|^{2}\right\}\right] x } \\
+ & \eta_{1}\left(\sum_{i=1}^{m}\left|\tilde{\xi}_{i}\right|\left|[P \mathcal{B}]_{i}\right|+\left|\vartheta_{i}\right|\left|[P \mathcal{B}]_{m+i}\right|\right) .
\end{aligned}
$$

By assumption, the quadratic term in $x$ is negative definite. The rest of the proof follows as for Theorem $5-c f$. (65).

Theorem 6 constitutes a fairly general statement, albeit at the cost of not giving explicit conditions on the control gains to ensure uniform global asymptotic stability. In the following proposition we restrict the choice of the control gains so as to make the constants $\eta_{i_{k}}$ in $\mathcal{A}$-see (72), equal to zero. Then, more tractable sufficient conditions for $\mathcal{A}$ to be Hurwitz follow. Proposition 1 Let $q_{d}$ be given as in Definition 5. Consider the system (42) and the output-feedback dynamic controller (45) 


$$
\begin{aligned}
& \mathcal{A}=\left[\begin{array}{ccccccccc}
-k_{p_{1}} & 1 & 0 & 0 & k_{d_{1}} & 0 & \cdots & \cdots & 0 \\
-\eta_{2_{1}} & -\left[k_{p_{2}}-k_{p_{1}}\right] & & & \mu_{2_{1}} & \ddots & & & \\
\vdots & -\eta_{3_{2}} & \ddots & & \vdots & \mu_{3_{2}} & & & \\
& & & 1 & & \vdots & & \ddots & 0 \\
-\eta_{m_{1}} & -\eta_{m_{2}} & \cdots & -\left[k_{p_{m}}-k_{p_{m-1}}\right] & \mu_{m_{1}} & \mu_{m_{2}} & \cdots & \mu_{m_{m-1}} & k_{d_{m}} \\
-k_{d_{1}} & 0 & \ldots & 0 & -a_{1} & 0 & \cdots & & 0 \\
b_{2} \eta_{2_{1}} & -k_{d_{2}} & & & -b_{2} \mu_{2_{1}} & \ddots & & & \vdots \\
\vdots & b_{3} \eta_{3_{2}} & \ddots & & \vdots & -b_{3} \mu_{3_{2}} & & & \\
& & & 0 & & \vdots & & \ddots & 0 \\
b_{m} \eta_{m_{1}} & b_{m} \eta_{m_{2}} & \cdots & -k_{d_{m}} & -b_{m} \mu_{m_{1}} & -b_{m} \mu_{m_{2}} & \cdots & -b_{m} \mu_{m_{m-1}} & -a_{m}
\end{array}\right] \otimes I_{n \times n}
\end{aligned}
$$

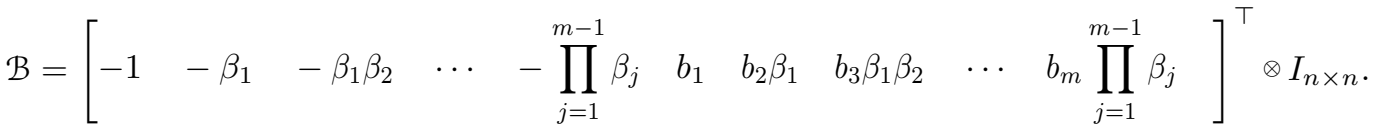

and (68) under Assumptions 1 and 2. Consider the expressions (58) and (71) and let the control gains be such that (77) holds,

$$
\begin{gathered}
\{m \geq 2\} \quad \Rightarrow \quad k_{p_{1}}=k_{d_{1}} \\
k_{p_{i}}=\frac{k_{p_{i-1}}+\left[k_{p_{i-1}}^{2}+4 k_{d_{i}}^{2}\right]^{1 / 2}}{2} \\
\forall i \in[2, m-1], m \geq 3
\end{gathered}
$$

and, for all $i \in[1, m]$ and $m \geq 2$,

$$
\min \left\{\frac{a_{i}}{b_{i}^{2}}, k_{p_{i}}+\operatorname{sgn}(1-i) k_{p_{i-1}}\right\}>\left[\eta_{2}^{2}+\eta_{3}^{2}\right]\left(\prod_{j=0}^{i-1} \beta_{j}\right)^{2},
$$

where $\operatorname{sgn}(0)=0$ and $\beta_{0}:=1$. If moreover the matrix $Q$ on top of next page is such that $Q-1 / 2 \operatorname{diag}\{Q\}$ is positive semidefinite, the origin is uniformly globally asymptotically stable.

The definition of $k_{p_{i}}$ in (83) solves the equations $\eta_{i_{k}}=0$, according to (72), for all applying $i, k \leq m=3$. In other words, condition (83b) is not needed if $m<3$. Then, we can set $P=I$ and, consequently, $Q=-\left(\mathcal{A}^{\top}+\mathcal{A}\right)$. This matrix, which for ease of reference is written in the top of next page, is positive semidefinite if: (i) the shadowed block $Q_{1} \otimes I$ is positive definite, which holds if $k_{p_{i}}>k_{p_{i-1}}$ and $k_{p_{i}}\left[k_{p_{i}}-k_{p_{i-1}}\right]>1$ for all $i \leq m$; (ii) the shadowed block $Q_{3}{ }^{\otimes I}$ is positive definite for sufficiently large values of $a_{i}>$ $a_{i-1}$. Furthermore, the Schur complement of $Q_{3} \otimes I$ i.e., $\left[Q_{1}-\right.$ $\left.Q_{2}^{\top} Q_{3}^{-1} Q_{2}\right] \otimes I$, is positive for sufficiently large values of $a_{i}$, $i \leq m$, that is, if $Q$ is diagonal-dominant. One may draw the same conclusion for $Q-1 / 2 \operatorname{diag}\{Q\}$ by enforcing the lower bounds on $a_{i}$ and $k_{p_{i}}$. The condition (84) implies that

$$
\frac{1}{2} \operatorname{diag}\{Q\}>\left[\eta_{2}^{2}+\eta_{3}^{2}\right] \operatorname{diag}\left\{\left|[P \mathcal{B}]_{i}\right|^{2}\right\}
$$

with $P=I_{2 m n \times 2 m n}-c f$. Ineq. (78).

Note, from (71), that $\beta_{j}$ depends on $k_{d_{j}}, k_{p_{j}}$ and $b_{j}$ therefore, the right-hand side of (84) depends on the control gains indexed up to $i-1$ only. Thus, by a suitable choice of $k_{p_{i}}$, according to (83b), the latter and (84) may be met simultaneously.
Proof of Proposition 1. Since in view of (83b) we have $\eta_{i_{k}}=$ 0 for all applying $i$ and $k$ the derivative of $W=|x|^{2}$ yields, using $Q-1 / 2 \operatorname{diag}\{Q\} \geq 0$ and (81),

$$
\begin{aligned}
\dot{W} \leq- & \left(\sum_{i=1}^{m}\left[k_{p_{i}}-k_{p_{i-1}}\right]\left|\tilde{\xi}_{i}\right|^{2}+a_{i}\left|\vartheta_{i}\right|^{2}\right)-k_{p_{0}}\left|\tilde{\xi}_{i}\right|^{2} \\
& -2\left(\sum_{i=1}^{m} \prod_{j=0}^{i-1} \beta_{j} \tilde{\xi}_{i}^{\top}-b_{i} \prod_{j=0}^{i-1} \beta_{j} \vartheta_{i}^{\top}\right)\left[\dot{\xi}_{1}^{*}-\dot{\xi}_{1 \circ}^{*}\right]
\end{aligned}
$$

Furthermore, considering once more (58), we obtain

$$
\begin{aligned}
{\left[\tilde{\xi}_{i}+b_{i} \vartheta_{i}\right]^{\top}\left[\dot{\xi}_{1}^{*}-\dot{\xi}_{1 \circ}^{*}\right] } & \leq \frac{1}{2} \sum_{i=1}^{m}\left[\eta_{2}^{2}+\eta_{3}^{2}\right]\left(\left|\tilde{\xi}_{i}\right|^{2}+b_{i}^{2}\left|\vartheta_{i}\right|^{2}\right) \\
& +\eta_{1}\left(\left|\tilde{\xi}_{i}\right|+\left|\vartheta_{i}\right|\right)+\left[|\dot{\tilde{q}}|^{2}+\left|\vartheta_{0}\right|^{2}\right] m
\end{aligned}
$$

hence, in view of (84) we conclude that there exist positive constants $\alpha_{1_{i}}, \alpha_{2_{i}}$ for all $i \in\{1 \ldots, m\}$, such that the total derivative of

$$
\mathcal{V}=\frac{1}{2}\left(\dot{\tilde{q}}^{\top} D\left(\tilde{q}+q_{d}(t)\right) \dot{\tilde{q}}+k_{p_{0}}|\tilde{q}|^{2}+\frac{k_{d_{0}}}{b_{0}}\left|\vartheta_{0}\right|^{2}\right)+\frac{1}{2}|x|^{2}
$$

along the closed-loop trajectories of (49), (76) satisfies

$$
\begin{aligned}
\dot{\mathcal{V}} \leq- & \frac{1}{2}\left(\sum_{i=1}^{m} \alpha_{1_{i}}\left|\tilde{\xi}_{i}\right|^{2}+\alpha_{2_{i}}\left|\vartheta_{i}\right|^{2}\right)+\eta_{1}\left(\left|\tilde{\xi}_{i}\right|+\left|\vartheta_{i}\right|\right) \\
- & {\left[\frac{a_{0} k_{d_{0}}}{b_{0}}-m\right]\left|\vartheta_{0}\right|^{2}+\left[k_{c} k_{\delta}+\frac{m+2}{2}\right]|\dot{\tilde{q}}|^{2} }
\end{aligned}
$$

$-c f$. Ineq. (65). The rest of the proof follows as for Theorem 5 .

An important corollary, for the case of $m=2$, stems from Proposition 1: a statement on uniform global asymptotic stability under output feedback for robot manipulators with flexible joints -Eq. (44). This corollary is another significant contribution to the theory of robot control since we are not aware of any similar result. Indeed, it must be recalled that very few results exist on position feedback tracking control of flexible-joint manipulators, one of the first of this nature is [41]. In the article [42] the same authors present a global 


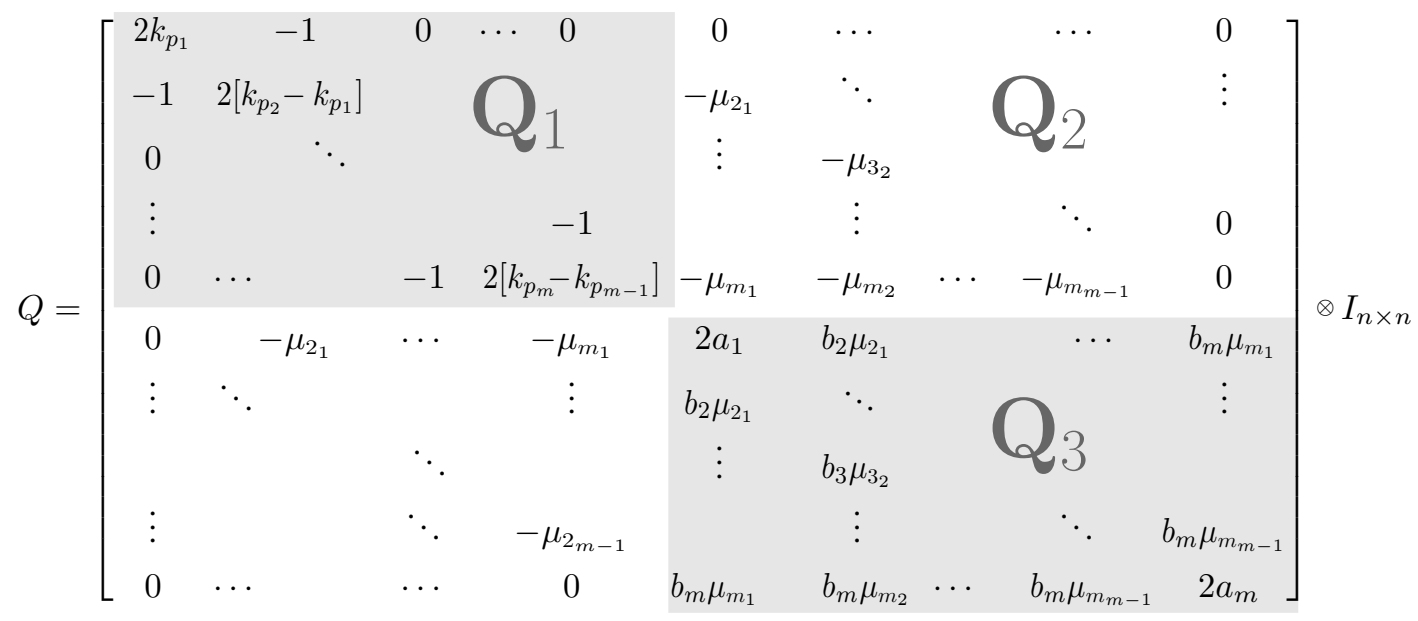

result using link positions and velocities. The controller in the following corollary makes use of actuator velocity measurements $\dot{q}_{2}$ but not of link velocities nor, of course, any other higher derivative (accelerations, jerks, etc).

Corollary 3 (Flexible-joint manipulators) Let $q_{1 d}$ be a given reference trajectory satisfying Definition 5. Consider the system (44) under Assumptions 1 and 2 with $g\left(q_{1}\right)=$ $\mathrm{g}\left(q_{1}\right)+K q_{1}$ in closed-loop with the controller

$$
\tau=K\left(q_{2}-q_{1}\right)+J K^{-1} u,
$$

Equations (68) with $m=2$,

$$
\xi_{1}^{*}=-k_{p_{0}} \tilde{q}-k_{d_{0}} \vartheta_{0}+D\left(q_{1}\right) \ddot{q}_{1 d}+C\left(q_{1}, \dot{q}_{1 d}\right) \dot{q}_{1 d} g\left(q_{1}\right)
$$

Let $k_{p_{0}}, k_{d_{0}}$ satisfy (77) with $m=2$. Furthermore, define $k_{d_{1}}=k_{p_{1}}, \mu=k_{p_{1}}\left[k_{p_{1}}+a_{1}\right]$ and $\beta=k_{p_{1}}\left(1+b_{1}\right)$ and assume that

$$
\begin{array}{r}
\min \left\{k_{p_{1}}, \frac{\left[k_{p_{2}}-k_{p_{1}}\right]}{\beta^{2}}, \frac{a_{1}}{b_{1}^{2}}, \frac{a_{2}}{b_{2}^{2} \beta^{2}}\right\}>\left[\eta_{2}^{2}+\eta_{3}^{2}\right] \\
{\left[k_{p_{2}}-k_{p_{1}}\right] \geq \max \left\{\frac{2}{k_{p_{1}}}, \frac{4 \mu^{2}}{a_{1}}\right\}, \quad a_{1} a_{2} \geq 2 b_{2}^{2} \mu^{2},}
\end{array}
$$

Under these conditions, the origin of the closed-loop system is uniformly globally asymptotically stable.

Proof: The definition $k_{p_{1}}=k_{d_{1}}$ implies that $\eta_{2_{1}}=0$ -see (72). That is, the condition (83a) holds and (83b) does not apply since $m=2$. The condition (88) implies (84) with $i \in\{1,2\}$ hence, also (85) with

$$
Q=\left[\begin{array}{cccc}
2 k_{p_{1}} & -1 & 0 & 0 \\
-1 & 2\left[k_{p_{2}}-k_{p_{1}}\right] & -\mu & 0 \\
0 & -\mu & 2 a_{1} & -b_{2} \mu \\
0 & 0 & -b_{2} \mu & 2 a_{2}
\end{array}\right] \otimes I_{n \times n}
$$

Moreover, we see that $Q-1 / 2 \operatorname{diag}\{Q\}$ is positive semidefinite if so are

$\left[\begin{array}{cc}k_{p_{1}} & -1 \\ -1 & 1 / 2\left[k_{p_{2}}-k_{p_{1}}\right]\end{array}\right],\left[\begin{array}{cc}1 / 2\left[k_{p_{2}}-k_{p_{1}}\right] & -\mu \\ -\mu & 1 / 2 a_{1}\end{array}\right],\left[\begin{array}{cc}1 / 2 a_{1} & -b_{2} \mu \\ -b_{2} \mu & a_{2}\end{array}\right]$

which conditions hold in view of (89).

\section{DISCUSSION AND OPEN PROBLEMS}

Conceptually, the controller (15) may be regarded as composed of two parts: a set-point control law of the type "Proportional-Derivative with gravity cancellation" (similar, e.g., to that from [10]) which is passivity-based, and a second part which plays the role of a "feedforward" :

$$
v:=D(q) \ddot{q}_{d}+C\left(q, \dot{q}_{d}\right) \dot{q}_{d}+k_{p} q_{d} .
$$

Then, the control input (15c) may be re-written as

$$
u=-k_{p} q-k_{d} \vartheta+g(q)+v
$$

and the closed-loop system takes the form

$$
\dot{x}=F(x)+G(x) w, \quad x:=\left[q^{\top} \dot{q}^{\top} \vartheta^{\top}\right]^{\top}
$$

where $w=\left[\begin{array}{lll}0 & v^{\top} & \dot{q}_{d}^{\top}\end{array}\right]^{\top}$,

$F:=\left[\begin{array}{c}\dot{q} \\ D^{-1}\left[-C \dot{q}-k_{p} q-k_{d} \vartheta\right] \\ -a \vartheta+b \dot{q}\end{array}\right], G(x):=\left[\begin{array}{ccc}0 & 0 & 0 \\ 0 & D^{-1} & 0 \\ 0 & 0 & -b\end{array}\right]$.

The equation $\dot{x}=F(x)$ corresponds to that of the closed-loop system with the passivity-based set-point controller from [10], for the particular set-point reference $q_{d}=0$. Furthermore, the total time-derivative of the storage function

$$
V(q, \dot{q}, \vartheta)=\frac{1}{2}\left(\dot{q}^{\top} D(q) \dot{q}+k_{p}|q|^{2}+\frac{k_{d}}{b}|\vartheta|^{2}\right)
$$

along the trajectories of (92), yields

$$
\dot{V}=-\frac{k_{d} a}{b}|\vartheta|^{2}-\vartheta^{\top} b \dot{q}_{d}+\dot{q}^{\top} v
$$

from which we see that, since $b \dot{q}_{d}$ is bounded, for any given positive number $\lambda$ we have

$$
|\vartheta| \geq \lambda \Longrightarrow \dot{V} \leq-\left(\frac{k_{d} a}{b}-\frac{b k_{\delta}^{2}}{\lambda}\right)|\vartheta|^{2}+\dot{q}^{\top} v
$$

The latter may be interpreted as the map $v \mapsto \dot{q}$ being passive for "large" values of the filter output $\vartheta$. On the other hand, the dirty-derivatives filter (15a), (15b) defines an output strictly

\footnotetext{
${ }^{5}$ The use of quotes is motivated by the fact that $v$ depends on the states $q$.
} 
passive map $\dot{\tilde{q}} \mapsto \vartheta$ with finite $\mathcal{L}_{2}$ and DC gains. This reasoning leads, at least at an intuitive level, to recognize that if the outputs of the filter grow then, the feedback-interconnected system "becomes" passive hence, input-output stable. Such is the underlying idea in the proof of boundedness (Lemma 2). Formal statements and even appropriate definitions to describe these properties formally from an input-output viewpoint are missing at this point.

On another note, we emphasize that a key feature of the controller (15) is that it guarantees uniform global asymptotic stability which in turn implies total stability, that is, robustness with respect to bounded disturbances. This concept, introduced in [43], is better known in modern literature as local inputto-state stability. Establishing global input-to-state stability via a (strict) Lyapunov function remains an open challenge, both for Lagrangian systems under output-feedback and, more generally, for nonlinear time-varying systems.

In the context of the "global tracking problem" for robot manipulators, a remarkable paper pursuing this direction is [5]. Not underestimating the authors' contribution it must nevertheless be recognized that the main result in the latter reference is stated for systems with friction, i.e., given by (2). Indeed, a direct computation shows that by applying

$$
u=-k_{p} q-k_{d} \vartheta+g(q)+F \dot{q}_{d}+v
$$

to (2) we obtain the "power" balance equation

$$
\dot{V} \leq-\frac{k_{d} a}{b}|\vartheta|^{2}-\left(F-k_{c} k_{\delta}\right)|\dot{\tilde{q}}|^{2}+\left[\begin{array}{ll}
0 & \dot{\tilde{q}}^{\top} \vartheta^{\top}
\end{array}\right]^{\top} w
$$

which is in clear contrast with (22). The closed-loop system clearly defines an output strictly passive map $w \mapsto\left[\begin{array}{ll}0 & \dot{\tilde{q}}^{\top} \vartheta^{\top}\end{array}\right]$ for sufficiently large $F$. However, subject to $w=0$, we have $\dot{V} \leq 0$ so $V$ does not qualify as an input-to-state-stability Lyapunov function. Nonetheless, the authors of [5] smartly establish input-to-state stability under output feedback.

In that regard, note that input-to-state stability based on so-called "Lyapunov functions satisfying Lasalle's conditions" has been studied, at least since the milestone paper [33], where the main result is also motivated by a robot control problem. The topic is also formally treated in considerable depth in [4] and a number of references therein. Roughly speaking, in [33] it is established that a time-invariant system

$$
\dot{x}=f(x, w) \quad x:=\left[x_{1}^{\top} x_{2}^{\top}\right]^{\top} \in \mathbb{R}^{m}
$$

with input $w$, admits an input-to-state-stability Lyapunov function $V$ (positive definite and proper) such that

$$
\frac{\partial V}{\partial x} f(x, w) \leq-\alpha_{1}(|x|)+\gamma(|w|), \quad \alpha_{1} \in \mathcal{K}_{\infty}, \gamma \in \mathcal{K}
$$

provided that:

- there exist positive-definite proper functions $V_{1}$ and $V_{2}$, as well as class $\mathcal{K}$ functions $\alpha_{11}, \alpha_{21}, \alpha_{22}, \gamma_{1}$ and $\gamma_{2}$, such that

$$
\begin{gathered}
\frac{\partial V_{1}}{\partial x} f(x, w) \leq-\alpha_{11}\left(\left|x_{2}\right|\right)+\gamma_{1}(|w|) \\
\frac{\partial V_{2}}{\partial x} f(x, w) \leq-\alpha_{21}(|x|)+\alpha_{22}\left(\left|x_{2}\right|\right)+\gamma_{2}(|w|) ;
\end{gathered}
$$

- the functions $\alpha_{22}$ and $\alpha_{11}$ have the same order of growth.

The first property i.e., the existence of $V_{1}$ satisfying (97), in [33] is called quasi-input-to-state stability. The prefix quasi is motivated by the negative semi definiteness of $\dot{V}_{1}$ when $w \equiv 0$. The second property is referred to as input-output-tostate stability with output $x_{2}$ and it is a notion of detectability for nonlinear systems. A direct comparison of (95) and (97) reveals that the latter two have the same form. This is not the case for (94) since the arguments of the corresponding $\alpha_{11}$ and $\alpha_{12}$ are different.

Thus, as far as we know, the state of the art in constructing Lyapunov functions for nonlinear time-varying systems relies on functions that "satisfy Lasalle-like conditions" such as (97). The construction of an input-to-state-stability Lyapunov function $V$ for systems satisfying

$$
\left|x_{1}\right| \geq \lambda \Longrightarrow \frac{\partial V}{\partial x} f(x, w) \leq-\alpha_{1}\left(\left|x_{1}\right|\right)+\alpha_{2}\left(\left|x_{2}\right|\right) \gamma(|w|),
$$

as is the case of $V$ in (94), is, in our humble opinion, a challenging and interesting open problem. Inspired by the rationale of proof of Lemma 2, a good start seems to impose a finite input-output gain relation between $x_{1}$ and $x_{2}$.

\section{CONCLuSions}

We established a constructive proof of uniform global asymptotic stability Lagrangian systems without dissipative forces in closed loop with an observerless dynamic output feedback controller. Our technical results close a significant chapter on output feedback control of nonlinear systems and formalize the very intuitive conjecture that the damping necessary to stabilize the system may be effectively injected through a passive filter, even for high-order systems.

We believe that our findings may pave the way towards a simple observer-less dynamic output feedback control approach inspired by the backstepping method but avoiding the often-resulting cumbersome highly nonlinear control laws. On the grounds of systems' analysis, we have briefly sketched new challenging open problems on construction of strict Lyapunov functions for systems satisfying Lasalle's conditions modulo the gain of a passive filter. Research in these directions is currently pursued.

\section{ACKNOWLEDGMENTS}

The author greatly acknowledges the excellent handling of this paper by AE A. Papachristodoulou; he feels in debt to the reviewers for their patience and abundant technical comments which led to several corrections and modifications. The author shall always feel deeply grateful to his former $\mathrm{PhD}$ advisor $\mathrm{R}$. Ortega; he gave the "global-tracking" control problem as $\mathrm{PhD}$ subject to the author in 1993. The technical contents of this paper is based on the little that the author has learned from the lot that E. Panteley has taught him during twenty years of joint work. 


\section{REFERENCES}

[1] E. Zergeroglu, D. M. Dawson, M. S. de Queiroz, and M. Krstić, "On global output feedback tracking control of robot manipulators," in Proc. 39th. IEEE Conf. Decision Contr., (Sydney, Australia), pp. 5073-5078, 2000.

[2] F. Mazenc, L. Praly, and W. P. Dayawansa, "Global stabilization of output feedback: examples and counterexamples," Syst. \& Contr. Letters, vol. 22, pp. 119-125, 1994.

[3] R. Marino and P. Tomei, "Dynamic output feedback linearization and global stabilization," Syst. \& Contr. Letters, vol. 17, no. 2, pp. 115-121, 1991.

[4] M. Malisoff and F. Mazenc, Constructions of Strict Lyapunov functions. London: Springer Verlag, 2009.

[5] E. V. L. Nunes and L. Hsu, "Global tracking for robot manipulators using a simple causal PD controller plus feedforward," Robotica, vol. 28, no. 1, pp. 23-34, 2010. DOI:10.1017/S0263574709005529.

[6] A. Loría, "Global tracking control of one degree of freedom EulerLagrange systems without velocity measurements," European J. of Contr., vol. 2, no. 2, 1996.

[7] E. Børhaug and K. Y. Pettersen, "Global output feedback pid control for n-dof euler-lagrange systems," in Proc. IEEE American Control Conference, (Minneapolis, Minnesota, USA), pp. 4993-4999, 2006.

[8] I. V. Burkov, "Mechanical system stabilization via differential observer," in IFAC Conference on System Structure and Control, (Nantes, France), pp. 532-535, 1995.

[9] A. Loría and R. Ortega, "On tracking control of rigid and flexible joints robots," Appl. Math. and Comp. Sci., vol. 5, no. 2, pp. 101-113, 1995.

[10] R. Kelly, "A simple set-point robot controller by using only position measurements," in Proc. 12th. IFAC World Congress, vol. 6, (Sydney, Australia), pp. 173-176, 1993.

[11] H. Berghuis and H. Nijmeijer, "Global regulation of robots using only position measurements," Syst. \& Contr. Letters, vol. 21, pp. 289-293, 1993.

[12] A. Jain and G. Rodriguez, "Diagonalized Lagrangian robot dynamics," IEEE Trans. on Robotics Automat., vol. 11, no. 4, pp. 571-584, 1995.

[13] G. Besançon, Contributions à l'étude et à l'observation des systèmes non linéaires avec recours au calcul formel. PhD thesis, INPG, 1996.

[14] M. Mabrouk, Transformation des systèmes Euler-Lagrange, observabilité et systèmes discrets. $\mathrm{PhD}$ thesis, Université Paul Verlaine-Metz, Metz, France, 2006. In French.

[15] I. Sarras, R. Ortega, and E. Panteley, "Asymptotic stabilization of nonlinear systems via sign-indefinite damping injection," in Proc. 51st. IEEE Conf. Decision Contr., pp. 2964-2969, Dec 2012.

[16] I. Sarras, On the constructive control and observer design of mechanical systems via passivity, immersion and invariance. $\mathrm{PhD}$ thesis, Université Paris-Sud XI, Orsay, France, 2010.

[17] J. G. Romero, Robust Energy Shaping Control of Nonlinear Systems. $\mathrm{PhD}$ thesis, Univ Paris Sud, Feb. 2013.

[18] G. Besançon, "Global output feedback tracking control for a class of Lagrangian systems," Automatica, vol. 36, no. 12, pp. 1915-1921, 2000.

[19] M. W. Spong, "Remarks on robot dynamics:canonical transformations and Riemannian geometry," in Proc. IEEE Conf. Robotics Automat., (Nice, France), pp. 554-559, 1992.

[20] M. Mabrouk, "Triangular form for Euler-Lagrange systems with application to the global output tracking control," Nonlinear Dynamics, vol. 60, no. $1-2$, pp. $87-98,2010$.

[21] M. Mabrouk and J.-C. Vivalda, "State transformation for Euler-Lagrange systems," in International Conference on Informatics in Control, $\mathrm{Au}$ tomation and Robotics, (Barcelona, Spain), pp. 43-48, 2005.

[22] J. G. Romero-Velázquez, I. Sarras, and R. Ortega, "A globally exponentially stable tracking controller for mechanical systems using position feedback," in Proc. IEEE American Control Conference, pp. 4976-4981, 2013.

[23] A. Astolfi, R. Ortega, and A. Venkatraman, "A globally exponentially convergent immersion and invariance speed observer for mechanical systems with non-holonomic constraints," Automatica, vol. 46, no. 1, pp. 182-189, 2010.

[24] A. Loria, "Uniform global position feedback tracking control of mechanical systems," in Proc. IEEE American Control Conference, (Washington, D.C.), pp. 5722-5727, 2013. DOI: 10.1109/ACC.2013.6580734.

[25] A. Loría, On output feedback control of Euler-Lagrange systems/Commande par retour de sortie des systèmes Euler-Lagrange. Thèse de doctorat label européen. $\mathrm{PhD}$ thesis, Université de Technologie de Compiègne, Compiègne, France, 7 Novembre 1996.

[26] W. Hahn, Stability of motion. New York: Springer-Verlag, 1967.
[27] A. R. Teel and L. Zaccarian, On the Literature's Two Different Definitions of Uniform Global Asymptotic Stability for Nonlinear Systems, vol. 328 of Lecture Notes in Control and Information Sciences, ch. in Advanced topics in control systems theory, pp. 285-289. DOI: 1-84628313-2. A. Loría, F. Lamnabhi-Lagarrigue, E. Panteley, eds., London: Springer Verlag, 2006.

[28] E. Panteley, A. Loría and A. Teel, "Relaxed persistency of excitation for uniform asymptotic stability," IEEE Trans. on Automat. Contr., vol. 46, no. 12 , pp. $1874-1886,2001$.

[29] R. Ortega, A. Loría P. J. Nicklasson, and H. Sira-Ramírez, Passivitybased Control of Euler-Lagrange Systems: Mechanical, Electrical and Electromechanical Applications. Series Comunications and Control Engineering, Springer Verlag, London, 1998. ISBN 1-85233-016-3.

[30] T. I. Fossen, Guidance and control of ocean vehicles. John Wiley \& Sons Ltd., 1994.

[31] F. Ghorbel, B. Srinivasan, and M. W. Spong, "On the uniform boundedness of the inertia matrix of serial robot manipulators," J. of Robotic Systems, vol. 151, pp. 17-28, 1998.

[32] R. Gunawardana and F. Ghorbel, "On the uniform boundedness of the coriolis/centrifugal terms in the robot equations of motion," Int. J. Robot. Automat., vol. 14, no. 2, pp. 45-53, 1999.

[33] D. Angeli, "Input-to-State Stability of PD-controlled robotic systems," Automatica, vol. 35, no. 7, pp. 1285-1290, 1999.

[34] A. Loría, "Observer-less output feedback global tracking control of lossless Lagrangian systems.” e-print no. arXiv:1307.4659, June 2013. Available from http://arxiv.org/abs/1307.4659

[35] E. Panteley, "Personal correspondance "on the growth of the solutions of Euler-Lagrange systems with global-lipschitz controls"." Available upon request. panteley@lss.supelec.fr, Feb. 2015

[36] R. Marino and P. Tomei, "Global adaptive output feedback control of nonlinear systems. Part I : Linear parameterization," IEEE Trans. on Automat. Contr., vol. 38, pp. 17-32, 1993.

[37] I. V. Burkov and A. T. Zaremba, "Dynamics of elastic manipulators with electric drives," Izv. Akad. Nauk SSSR Mekh. Tverd. Tela, vol. 22, no. 1, pp. 57-64, 1987. Engl. transl. in Mechanics of Solids, Allerton Press.

[38] M. Spong, "Modeling and control of elastic joint robots," ASME J. Dyn. Syst. Meas. Contr., vol. 109, pp. 310-319, 1987.

[39] E. Panteley and A. Loría, "On global uniform asymptotic stability of non linear time-varying non autonomous systems in cascade," Syst. \& Contr. Letters, vol. 33, no. 2, pp. 131-138, 1998.

[40] R. Gunawardana and F. Ghorbel, "On the boundedness of the Hessian of the potential energy of robot manipulators," J. of Robotic Systems, vol. 16, no. 11, pp. 613-625, 1999.

[41] S. Nicosia and P. Tomei, "A tracking controller for flexible joint robots using only link position feedback," IEEE Trans. on Automat. Contr. vol. 40, no. 5, pp. 885-890, 1995 .

[42] S. Nicosia and P. Tomei, "Output feedback control of flexible joint robots," in Proc. Int. Conf. on Systems, Man and Cybernetics, 'Systems Engineering in the Service of Humans', vol. 3, pp. 700-704, 1993.

[43] I. G. Malkin, "K voprosu ob obratimosti teoremi Liapunova ob asimptoticheskoi yctorchivosti," Prikl. Mat. i Mekh., vol. 18, pp. 129-138, 1954. In Russian. Usually cited in English as: On the reciprocal of Lyapunov's theorem on asymptotic stability.

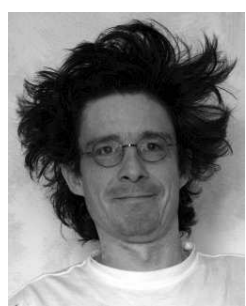

Antonio Loría was born in Mexico City in 1969. He got the BSc degree in Electronic Engineering from the ITESM, Monterrey, Mexico in 1991. He got the MSc and $\mathrm{PhD}$ degrees in Control Engg. from the UTC, France in 1993 and 1996 respectively. He is IEEE member since 1990. From December 1996 through Dec. 1998, he was successively an associate researcher at Univ. of Twente, The Netherlands; NTNU, Norway and the CCEC of the Univ. of California at Santa Barbara, CA, USA. A. Loria has the honour of holding a research position at the the French National Centre of Scientific Research (CNRS) since january 1999 (as "Directeur de Recherche" since 2006). His research interests include dynamical systems theory (stability, chaos), control systems theory (adaptive, output feedback, discrete-time, continuoustime, linear, non-linear, hybrid, robust, passive, etc.). Between 2003-2015 he served as associate editor for Systems and Control Letters, Automatica, IEEE Transactions on Automatic Control, IEEE Transactions on Control Systems Technology, Control Systems Magazine and Revista Iberoamericana de Automatica Industrial. He has also been a member of the IEEE CSS Conference Editorial Board and diverse conference IPCs for several years. To see further, please visit http://antonio.loria.12s.supelec.fr. 